TITLE:

Crossing Symmetry in Elliptic Solutions of the Yang-Baxter Equation and a New L-operator for Belavin's Solution

$\operatorname{AUTHOR}(S)$ :

HASEGAWA, Koji

CITATION:

HASEGAWA, Koji. Crossing Symmetry in Elliptic Solutions of the Yang-Baxter Equation and a New L-operator for Belavin's Solution. 数理解析研究所講究録 1992, 816: 82-110

ISSUE DATE:

1992-12

URL:

http://hdl.handle.net/2433/83106

RIGHT: 


\title{
Crossing Symmetry in Elliptic Solutions of the Yang-Baxter Equation and a New L-operator for Belavin's Solution
}

Koji HASEGAWA,Mathematical Institute, Tohoku University

\begin{abstract}
Investigated are some algebraic structures in elliptic solutions of the Yang-Baxter equations. We prove the crossing symmetry in Belavin's model as well as in the $A_{n-1}^{(1)}$ face model, and we construct a new family of L-operators for Belavin's R-matrix as an application.
\end{abstract}

\section{Introduction}

Recently many progresses have been made in the theory of two dimensional solvable statistical lattice models. Among them we will investigate here some algebraic structures in elliptic solutions of the Yang-Baxter equations (YBE). Namely, we show the crossing symmetry in Belavin's model [Be] as well as in Jimbo et al.'s $A_{n-1}^{(1)}$ face model [JKMO], and we construct a new family of L-operators for Belavin's model as an application.

In [BS] Bazhanov and Stroganov showed that the chiral Potts model, which is a solution of the YBE or the star-triangle relation whose spectral parameter lies in a high genus algebraic curve [AMPTY] [BPA], is a "descendant" of the 
6-vertex model which is nothing but the R-matrix associated to $U_{q}\left(\widehat{s l}_{n}\right)$. That is, they derived the chiral Potts model as the intertwiner of cyclic L-operators, or equivalently, the intertwiner of two-fold tensor of cyclic representations of $U_{q}\left(\hat{s l}_{n}\right)$. Motivated by their result, in our previous paper [HY] we have shown that Kashiwara-Miwa's elliptic solution (the so-called broken $\mathbf{Z}_{N}$ symmetric solution) $[\mathrm{KM}]$ is a descendant of Baxter's 8-vertex model [Bax] in the above sense : Take Sklyanin's cyclic L-operator for the 8-vertex model and we get Kashiwara-Miwa's model as the intertwiner for the L-operators. Along with this derivation, in $[\mathrm{H}]$ we further succeeded in relating the crossing symmetry of Kashiwara-Miwa's model with a certain duality property of the L-operators.

To generalize this story for the $n$-state elliptic model of Belavin, one immediately needs a cyclic L-operator for the model and its construction is one of our motivation here. We are inspired by an idea in Bazhanov et al. [BKMS]. They considered the $U_{q}\left(\widehat{s l}_{n}\right)$ generalization of [BS] by means of "intertwining vectors" or "factorized L-operators [IK]". Here intertwining vectors are originally appeared in [Bax73] to introduce face models via vertex models. Hence by definition they relate a vertex model and a certain face model, and using this relationship [BKMS] observed that a simple combination of intertwining vectors provides an L-operator. Intertwining vectors between Belavin's model and the $A_{n-1}^{(1)}$ face model are given in [JMO] and they are, so to speak, "outgoing" intertwining vectors. What we need more to construct L-operators are their "dual" or the "incoming" intertwining vectors and our method to construct them is as follows: we first observe the crossing symmetry of the models, which is nothing but the incoming/outgoing duality (sections 3 and 4 ), and then we obtain the incoming intertwining vectors by fusing [C][JKMO] the original intertwining vectors (section 5 ) . The resulting L-operators (section 6 ) act on $\mathbf{C}^{n} \otimes$ (space of functions on the weight space $\mathbf{h}^{*}$ of $s l_{n}$ ), and letting 
the deformation parameter $q=e^{\hbar}$ of Belavin's model to be a root of unity there arise invariant subspaces and we can get the desired cyclic L-operators. In addition to this cyclic one we can also find out other invariant subspaces so that we can generalize the analogue of Sklyanin's series of L-operators [S] for Baxter's 8-vertex model to the Belavin model.

As is well known, up to a certain transformation the trigonometric limit of Belavin's model gives the $R$ matrix of $U_{q}\left(\widehat{s l}_{n}\right)$ in the vector representation $[\mathrm{J}]$. In this sence what we have observed here can be regarded as a part of the theory of "elliptic" version of quantum groups [KRS] [KS], which we hope to discuss elsewhere.

\section{Review}

Belavin's vertex model [Be].

For $n>1$ let $\mathbf{C}^{n}=\oplus_{k=1}^{n} \mathbf{C} e^{k}$ and let $g, h \in G L\left(\mathbf{C}^{n}\right)$ to be $g e^{k}:=$ $e^{k} \exp \frac{2 \pi i k}{n}, h e^{k}:=e^{k+1}$ so that $g h=h g \exp \frac{2 \pi i}{n}$. Let $\hbar, \tau \in \mathrm{C}, \hbar \neq 0, \operatorname{Im} \tau>0$. Belavin's R-matrix is characterized as the unique solution of the following five conditions.

- $R(u)$ is a holomorphic $\operatorname{End}\left(\mathbf{C}^{n} \otimes \mathbf{C}^{n}\right)$-valued function in $u$,

- $R(u)=(x \otimes x) R(u)(x \otimes x)^{-1}$ for $x=g, h$,

- $R(u+1)=(g \otimes 1)^{-1} R(u)(g \otimes 1) \times(-1)$,

- $R(u+\tau)=(h \otimes 1) R(u)(h \otimes 1)^{-1} \times\left(-\exp 2 \pi i\left(u+\frac{\hbar}{n}+\frac{\tau}{2}\right)\right)^{-1}$,

- $R(0)=P: x \otimes y \mapsto y \otimes x$.

We also have the following formula for $R(u)[\mathrm{RT}]$ :

$$
R(u) e^{i} \otimes e^{j}=\sum_{i^{\prime}, j^{\prime}} e^{i^{\prime}} \otimes e^{j^{\prime}} R(u)_{i^{\prime} j^{\prime}}^{i j}
$$




$$
R(u)_{i^{\prime} j^{\prime}}^{i j}=\delta_{i+j, i^{\prime}+j^{\prime} \bmod n} \frac{\theta^{\left(i^{\prime}-j^{\prime}\right)}(u+\hbar)}{\theta^{\left(i^{\prime}-i\right)}(\hbar) \theta^{i-j^{\prime}}(u)} \frac{\prod_{k=0}^{n-1} \theta^{(k)}(u)}{\prod_{k=1}^{n-1} \theta^{(k)}(0)} .
$$

Here $\theta_{m, l}(u, \tau):=\sum_{\mu \in m+l \mathbf{Z}} \exp 2 \pi i\left(\mu u+\frac{\mu^{2}}{2 l} \tau\right)$ and $\theta^{(j)}(u):=\theta_{\frac{1}{2}-\frac{j}{n}, 1}\left(u+\frac{1}{2}, n \tau\right)$.

Then the YBE of the vertex type

$R^{23}\left(u_{2}-u_{3}\right) R^{13}\left(u_{1}-u_{3}\right) R^{12}\left(u_{1}-u_{2}\right)=R^{12}\left(u_{1}-u_{2}\right) R^{13}\left(u_{1}-u_{3}\right) R^{23}\left(u_{2}-u_{3}\right)$

holds, where $V_{i}$ are copies of $\mathbf{C}^{n}$ and $R^{i j}$ acts on $i$-th space and $j$-th space.

For the latter purpose we will reformulate this solution as follows. For each $u \in \mathbf{C}$ let $V\left(\square_{u}\right)$ be the copy of $\mathbf{C}^{n}$ and write the R-matrix $R(u-v)$ acting on $V\left(\square_{u}\right) \otimes V\left(\square_{v}\right)$ as $R\left(\square_{u}, \square_{v}\right)$. We also put $\check{R}^{\square_{u}, \square_{v}}:=P R^{\square_{u}, \square_{v}}$, where $P$ is the permutation $V\left(\square_{u}\right) \otimes V\left(\square_{v}\right) \rightarrow V\left(\square_{v}\right) \otimes V\left(\square_{u}\right)$. Then the YBE (2) reads as follows,

$$
\begin{aligned}
& \left(\check{R}^{\square_{v}, \square_{w}} \otimes 1\right)\left(1 \otimes \check{R}^{\square_{u}, \square_{w}}\right)\left(\check{R}^{\square_{u}, \square_{v}} \otimes 1\right) \\
= & \left(1 \otimes \check{R}^{\square_{u}, \square_{v}}\right)\left(1 \otimes \check{R}^{\square_{u}, \square_{w}}\right)\left(\check{R}^{\square_{v}, \square_{w}} \otimes 1\right) \\
& : V\left(\square_{u}\right) \otimes V\left(\square_{v}\right) \otimes V\left(\square_{w}\right) \rightarrow V\left(\square_{w}\right) \otimes V\left(\square_{v}\right) \otimes V\left(\square_{u}\right) .
\end{aligned}
$$

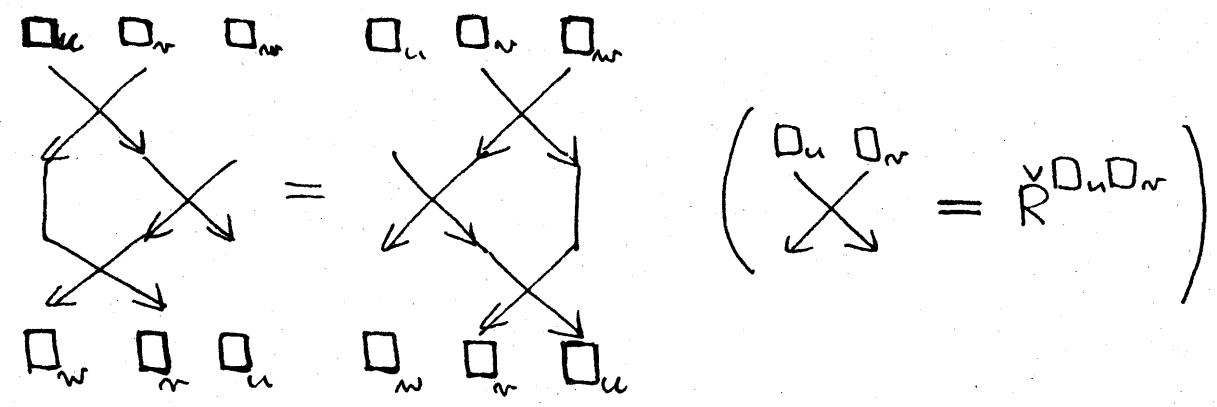

Remark. The notation $\square$ stands for the Young diagram consisting of one box. If we consider the 'algebra of L-operators' for Belavin's R-matrix then the notation $\square_{u}$ can be justified as its "vector representation with the spectral parameter $u "$.

$A_{n-1}^{(1)}$ face model [JMO]. 
Let $\epsilon_{i}(i=1, \cdots, n)$ be the orthonomal basis of an $n$ dimensional vector space with the inner product $($,$) and put \mathbf{h}^{*}:=\mathbf{C}$-Span of $\left\{\epsilon_{i}-\epsilon_{i+1}(i=1, \cdots, n-1)\right\}$ so that we can identify $\mathbf{h}^{*}$ and the weight space of the complex Lie algebra $s l_{n}$ in a usual way. Let $^{-}: \mathbf{C}^{n} \rightarrow \mathbf{h}^{*}$ be the orthogonal projection. Then the Boltzmann weight of the $A_{n-1}^{(1)}$ face model corresponding to the vector representation $\square$ is given by the following.

$$
\begin{aligned}
& \check{W}\left[\begin{array}{ccc} 
& \lambda+\bar{\epsilon}_{i} & \\
\lambda & u & \lambda+2 \bar{\epsilon}_{i} \\
& \lambda+\bar{\epsilon}_{i} &
\end{array}\right]:=\frac{h(u+\hbar)}{h(\hbar)} \\
& \check{W}\left[\begin{array}{cc}
\lambda+\bar{\epsilon}_{i} \\
\lambda \quad u & \lambda+\bar{\epsilon}_{i}+\bar{\epsilon}_{j} \\
\lambda+\bar{\epsilon}_{i} &
\end{array}\right]:=\frac{h\left(-u+\hbar \lambda_{i j}\right)}{h\left(\hbar \lambda_{i j}\right)},
\end{aligned}
$$

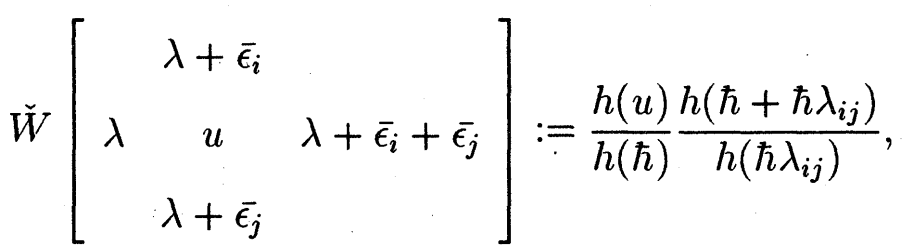

and for the other configulation of $\lambda, \mu, \mu^{\prime}$ and $\nu$

$$
\check{W}\left[\begin{array}{ccc} 
& \mu & \\
\lambda & u & \nu \\
& \mu^{\prime} &
\end{array}\right]:=0,
$$

where $h(u):=\theta_{1 / 2,1}(u+1 / 2, \tau)$ and

$$
\lambda_{i j}:=\left(\lambda+\rho, \bar{\epsilon}_{i}-\bar{\epsilon}_{j}\right), \rho \text { is the half sum of positive roots. }
$$

To formulate this weight as a linear operator or a 'face operator (an elementary transfer matrix)', the following vector space is in order.

$$
\mathcal{P}_{\lambda \square}^{\mu}: \cong \begin{cases}\mathbf{C} & : \mu=\lambda+\bar{\epsilon}_{i} \text { for some } i \\ 0 & : \text { otherwise }\end{cases}
$$


We denote by $e_{\lambda}^{\mu}$ the basis of the one dimensional space $\mathcal{P}_{\lambda \square}^{\mu}$ when $\mu=\lambda+\bar{\epsilon}_{i}$ for some $i$, and otherwise we set $e_{\lambda}^{\mu}=0$. For each $u \in \mathbf{C}$ we consider the copy $\mathcal{P}_{\lambda \square_{u}}^{\mu}$ of $\mathcal{P}_{\lambda \square}^{\mu}$ and define

$$
\begin{aligned}
& \mathcal{P}_{\lambda \square_{u_{1}} \cdots \square_{u_{k}}}^{\nu}:=\sum_{\mu_{1}, \cdots, \mu_{k-1}} \mathcal{P}_{\lambda \square_{u_{1}}}^{\mu_{1}} \otimes \mathcal{P}_{\mu_{1} \square_{u_{2}}}^{\mu_{2}} \otimes \cdots \otimes \mathcal{P}_{\mu_{k-1} \square_{u_{k-1}}}^{\nu} \\
& \mathcal{P}_{\square_{u_{1}} \ldots \square_{u_{k}}}:=\oplus_{\lambda, \nu} \mathcal{P}_{\lambda \square_{u_{1}} \ldots \square_{u_{k}}}^{\nu} .
\end{aligned}
$$

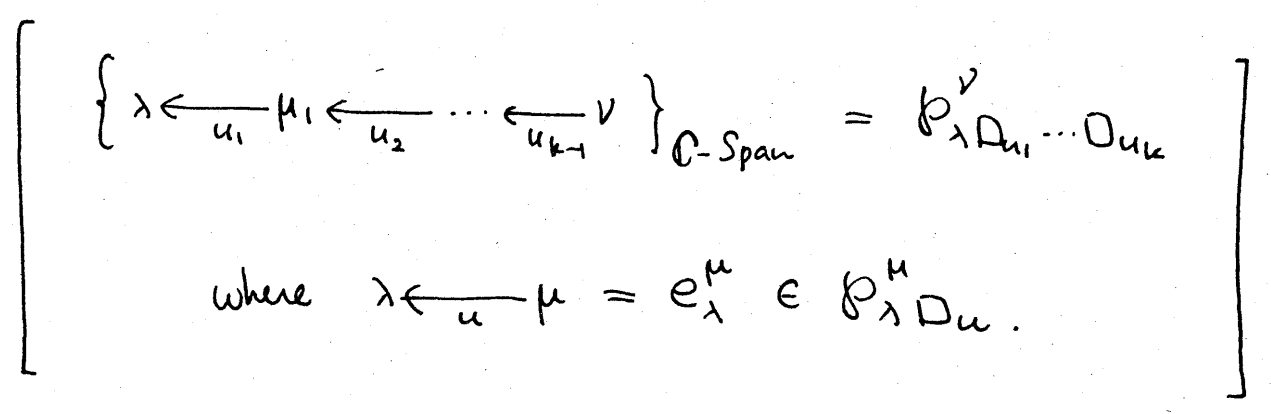

These are the space of "addmissible paths" in [JKMO]. For $e_{\lambda}^{\mu} \in \mathcal{P}_{\lambda \square_{u}}^{\mu}$ and $e_{\mu}^{\nu} \in \mathcal{P}_{\mu \square_{v}}^{\nu}$ we put

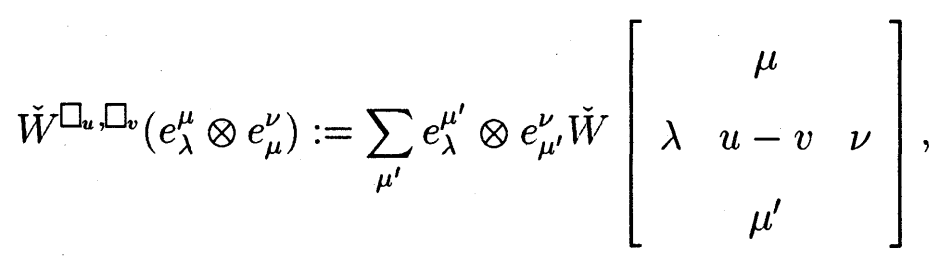
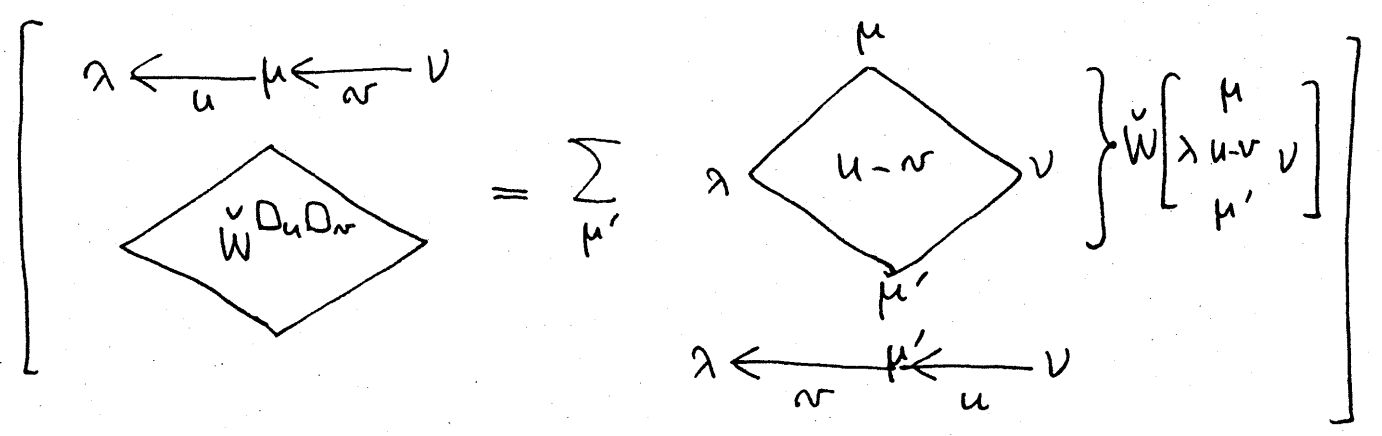

thereby define the face operator

$$
\check{W}^{\square_{u}, \square_{v}}: \mathcal{P}_{\square_{u} \square_{v}} \rightarrow \mathcal{P}_{\square_{v} \square_{u}}, \quad \mathcal{P}_{\lambda \square_{u} \square_{v}}^{\nu} \rightarrow \mathcal{P}_{\lambda \square_{v} \square_{u}}^{\nu}
$$


With these definitions the YBE of face type reads as follows: as operators $\mathcal{P}_{\nu \square_{u} \square_{v} \square_{w}}^{\lambda} \rightarrow \mathcal{P}_{\nu \square_{w} \square_{v} \square_{u}}^{\lambda}$ we have

$$
\begin{aligned}
& \left(1 \otimes \check{W}^{\square_{u}, \square_{v}}\right)\left(\check{W}^{\square_{u}, \square_{w}} \otimes 1\right)\left(1 \otimes \check{W}^{\square_{v}, \square_{w}}\right) \\
= & \left(\check{W}^{\square_{v}, \square_{w}} \otimes 1\right)\left(1 \otimes \check{W}^{\square_{u}, \square_{w}}\right)\left(\check{W}^{\square_{u}, \square_{v}} \otimes 1\right) .
\end{aligned}
$$
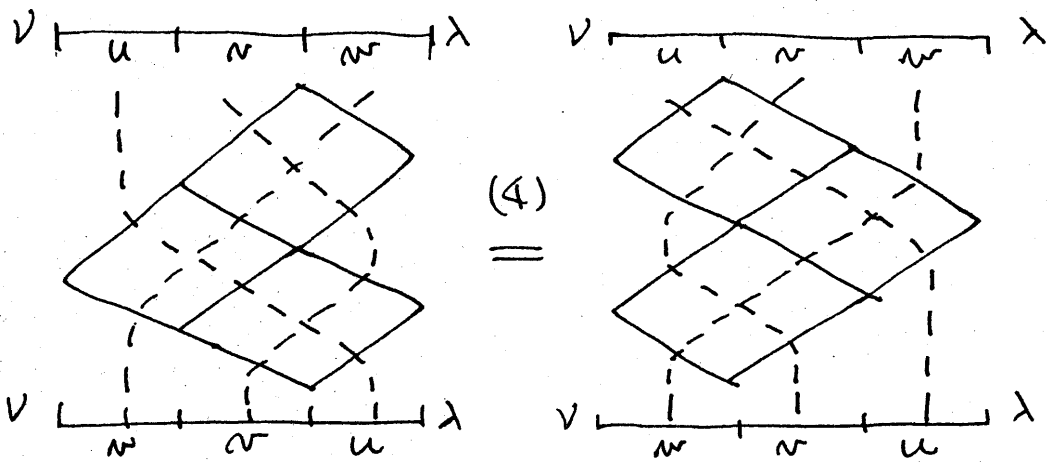

Intertwining vectors [JMO]. Put

$$
\left(\phi_{\lambda \square_{u}}^{\mu}\right)_{j}:=\left\{\begin{array}{cl}
\theta^{(j)}\left(u-n \hbar\left(\lambda+\rho, \overline{\epsilon_{k}}\right)\right) & : \mu-\lambda=\overline{\epsilon_{k}} \text { for some } k, \\
0 & : \text { otherwise }
\end{array}\right.
$$

and define the linear map

$$
\phi_{\lambda \square_{u}}^{\mu}: \mathcal{P}_{\lambda \square_{u}}^{\mu} \rightarrow V\left(\square_{u}\right)
$$

by

$$
\begin{aligned}
& \phi_{\lambda \square_{u}}^{\mu} e_{\lambda}^{\mu}:=\sum_{j} e^{j}\left(\phi_{\lambda \square_{u}}^{\mu}\right)_{j} .
\end{aligned}
$$

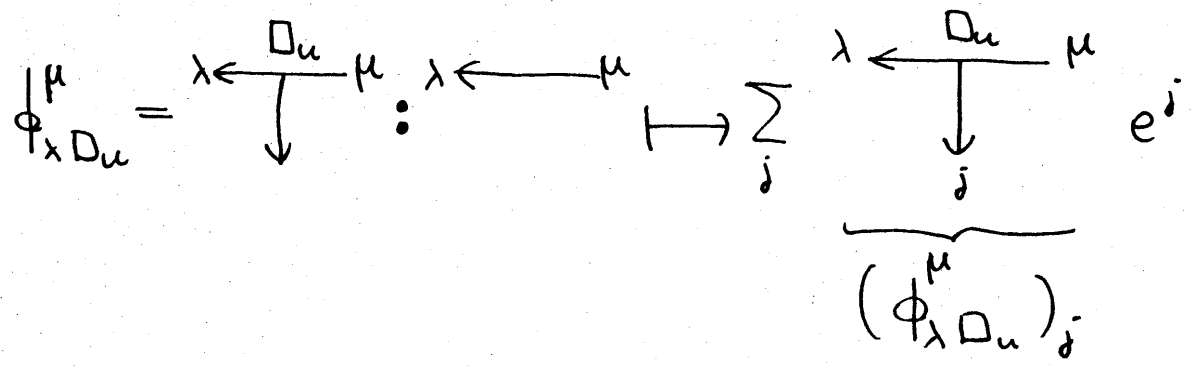

Then they "intertwine" Belavin's vertex model and the $A_{n-1}^{(1)}$ face model, 
namely

$$
\check{R}^{\square_{u}, \square_{v}} \phi_{\mu \square_{u}}^{\lambda} \otimes \phi_{\nu \square_{v}}^{\mu}=\sum_{\mu^{\prime}} \phi_{\mu^{\prime} \square_{v}}^{\lambda} \otimes \phi_{\nu \square_{u}}^{\mu^{\prime}} \check{W}\left[\begin{array}{c}
\mu \\
\lambda \\
u-v \\
\mu^{\prime}
\end{array}\right]
$$

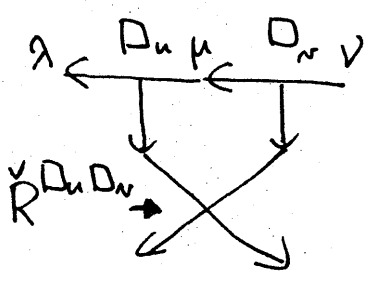

$\stackrel{(5)}{=} \sum_{\mu^{\prime}}$

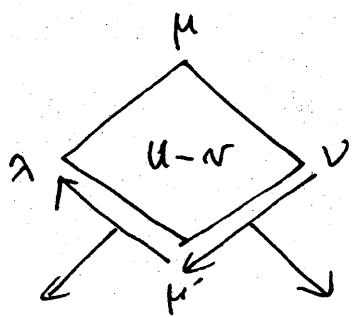

This formula is very remarkable because of its similarity between the monodoromy property of the $n$-point function in the $q$-conformal field theory [FR].

The quantity $\left\{\left(\phi_{\mu \square_{u}}^{\lambda}\right)_{j}\right\}_{j=1}^{n}$ regarded as an $n$-vector is called the intertwining vector.

\section{Crossing symmetry in the vertex models}

Fusion procedure[C]. Let

$$
\begin{gathered}
\check{R}^{\square_{u_{1}} \otimes \square_{u_{2}} \otimes \cdots \otimes \square_{u_{l}}, \square_{v}}:=\left(\check{R}^{\square_{u_{1}}, \square_{v}}\right)^{1,2}\left(\check{R}^{\square_{u_{2}}, \square_{v}}\right)^{2,3} \cdots\left(\check{R}^{\square_{u_{l}}, \square_{v}}\right)^{l, l+1}, \\
\check{R}^{\square_{u_{1}} \otimes \cdots \otimes \square_{u_{k}}, \square_{v_{1}} \otimes \cdots \otimes \square_{v_{l}}}:= \\
\left(\check{R}^{\square_{u_{1}} \otimes \cdots \otimes \square_{u_{k}}, \square_{v_{l}}}\right)^{k \cdots k+l-1 ; k+l} \cdots\left(\check{R}^{\square_{u_{1}} \otimes \cdots \otimes \square_{u_{k}}, \square_{v_{2}}}\right)^{2 \cdots k+1 ; k+2}\left(\check{R}^{\square_{u_{1}} \otimes \cdots \otimes \square_{u_{k}}, \square_{v_{1}}}\right)^{1 \cdots k ; k+1} .
\end{gathered}
$$




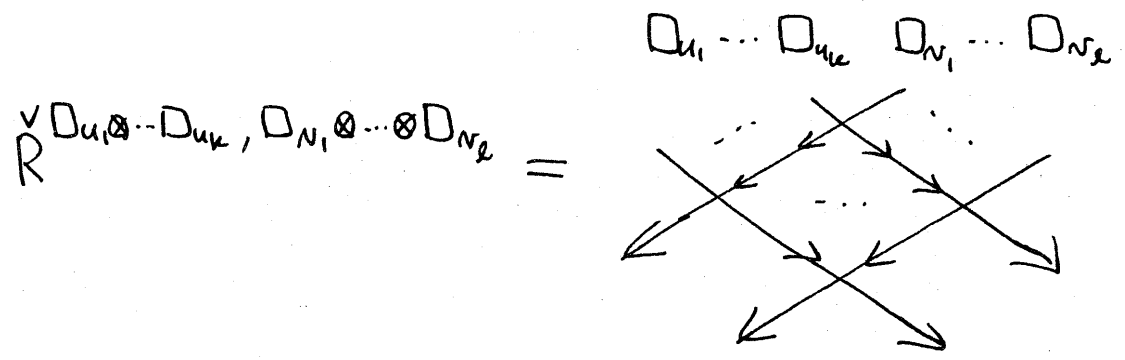

For $k=1, \cdots, n$ let $1^{k}$ be the Young diagram of vertical $k$ boxes $\left(1^{1}=\square\right.$ ; in this paper we will treat these special diagrams for simplicity.). Then the fusion operator by Cherednik associated with $1^{k}$ is given by

$$
\begin{gathered}
\pi_{1^{k}}: V\left(\square_{u}\right) \otimes \cdots \otimes V\left(\square_{u+(k-1) \hbar}\right) \rightarrow V\left(\square_{u+(k-1) \hbar}\right) \otimes \cdots \otimes V\left(\square_{u}\right) \\
:=\left(\check{R}^{\square_{u_{1}}, \square_{u_{2}}}\right)^{k-1 ; k} \cdots\left(\check{R}^{\square_{u_{1}} \otimes \square_{u_{2}} \otimes \cdots \otimes} \square_{u_{k-2}}, \square_{u_{k-1}}\right)^{2 \cdots k-1 ; k}\left(\check{R}^{\square_{u_{1}} \otimes \square_{u_{2}} \otimes \cdots \otimes \square_{u_{k-1}}, \square_{u_{k}}}\right)^{1 \cdots k-1 ; k},
\end{gathered}
$$

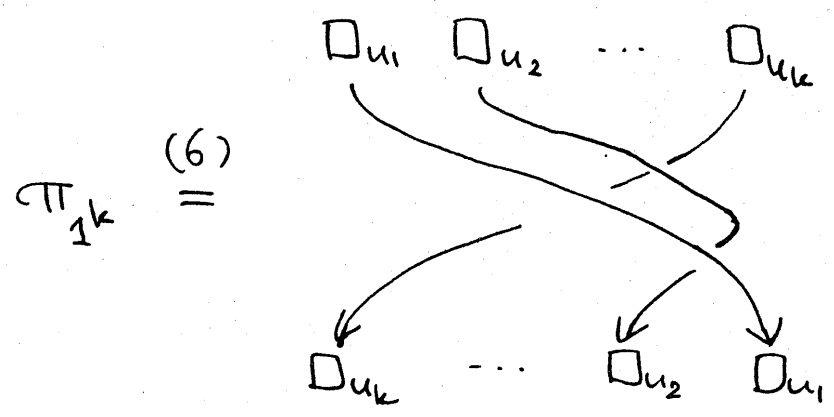

where the spectral parameters are specialized as

$$
\left(u_{1}, \cdots, u_{k}\right)=(u, u+\hbar, \cdots, u+(k-1) \hbar)
$$

so that the rank of the operator $\pi$ degenerates. By virtue of the YBE (4) the factors in (6) can be arranged in various ways by 'braid manipulation' and this is the key remark in deriving the formula in what follows. We denote the image of $\pi_{1^{k}}$ in $V\left(\square_{u+(k-1) \hbar}\right) \otimes \cdots \otimes V\left(\square_{u}\right)$ as $V\left(1_{u}^{k}\right)$ and then it turns out that $V\left(1_{u}^{k}\right)=\wedge^{k}\left(\mathbf{C}^{n}\right)$ for the generic value of $\hbar$. Put 


$$
\check{R}^{\mathbf{K}, \mathbf{L}}:=\left.\check{R}^{\square_{u+(k-1) \hbar} \otimes \cdots \otimes \square_{u}, \square_{v+(l-1) \hbar} \otimes \cdots \otimes \square_{v}}\right|_{V(\mathbf{K}) \otimes V(\mathbf{L})},
$$

where

$$
\mathbf{K}=1_{u}^{k}, \quad \mathbf{L}=1_{v}^{l}
$$

are shorthand notation. Then the YBE for $\check{R}^{\square_{u}, \square_{v}}$ (4) guarantees that this 'fused' operator preserves the image of $\pi$ 's

$$
\check{R}^{\mathbf{K}, \mathbf{L}}: V(\mathbf{K}) \otimes V(\mathbf{L}) \rightarrow V(\mathbf{L}) \otimes V(\mathbf{K})
$$

as well as they enjoy the YBE:

$$
\left(\check{R}^{\mathbf{L}, \mathbf{M}} \otimes 1\right)\left(1 \otimes \check{R}^{\mathbf{K}, \mathbf{M}}\right)\left(\check{R}^{\mathbf{K}, \mathbf{L}} \otimes 1\right)=\left(1 \otimes \check{R}^{\mathbf{K}, \mathbf{L}}\right)\left(\check{R}^{\mathbf{K}, \mathbf{M}} \otimes 1\right)\left(1 \otimes \check{R}^{\mathbf{L}, \mathbf{M}}\right)
$$
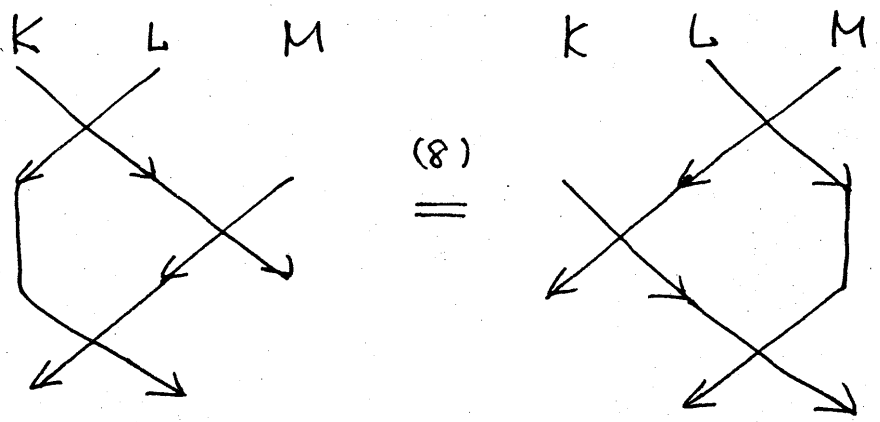

Crossing symmetry. Let us denote the special diagram $1^{n}$ as top and put

$$
\left(1_{w}^{m}\right)^{*}:=1_{w+m \hbar}^{n-m}
$$

Then since $\pi_{\text {top }}=\check{R}^{\mathbf{K}, \mathbf{K}^{*}}\left(\pi_{\mathbf{K}} \otimes \pi_{\mathbf{K}^{*}}\right)$ for each $\mathbf{K}=1_{u}^{k}$, we can define a pairing

$$
<,>: V(\mathbf{K}) \otimes V\left(\mathbf{K}^{*}\right) \rightarrow V\left(\operatorname{top}_{u}\right) \rightarrow \mathbf{C}
$$

as the composition of $\check{R}^{\mathbf{K}, \mathbf{K}^{*}}$ and the identification map $\mid t o p_{u}>\mapsto 1$, where 
$\mid t o p_{u}>$ is a fixed basis of the 1-dimensional space $V\left(t o p_{u}\right)$.

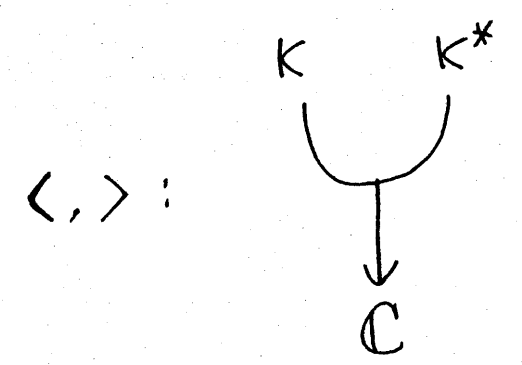

For generic $\hbar$ this pairing turns out to be non-degenarate so that we can and do identify $V(\mathbf{K})^{*}$ and $V\left(\mathbf{K}^{*}\right)$, where $V(\mathbf{K})^{*}$ stands for the dual space of $V(\mathbf{K})$. Fix $\mathbf{K}=1_{u}^{k}$ and $\mathbf{L}=1_{v}^{l}$. We take a basis $\left\{e^{I}\right\}_{I}$ in $V(\mathbf{K})$ and its dual basis (with respect to $<,>$ ) $\left\{e_{*}^{I}\right\}_{I}$ in $V\left(\mathbf{K}^{*}\right)$, and do the same for $\mathbf{L}$. We define the matrix elements of $\check{R}$ by

$$
\begin{array}{cl}
\check{R}^{\mathbf{K}, \mathbf{L}} e^{I} \otimes e^{J}=\sum_{I^{\prime}, J^{\prime}} e^{J^{\prime}} \otimes e^{I^{\prime}}\left(\check{R}^{\mathbf{K}, \mathbf{L}}\right)_{J^{\prime} I^{\prime}}^{I J} & \left(e^{I} \in V(\mathbf{K}), e^{J} \in V(\mathbf{L})\right), \\
\check{R}^{\mathbf{K}, \mathbf{L}^{*}} e^{I} \otimes e_{*}^{J}=\sum_{I^{\prime}, J^{\prime}} e_{*}^{J^{\prime}} \otimes e^{I^{\prime}}\left(\check{R}^{\mathbf{K}, \mathbf{L}^{*}}\right)_{J^{\prime} I^{\prime}}^{I J} & \left(e^{I} \in V(\mathbf{K}), e_{*}^{J} \in V\left(\mathbf{L}^{*}\right)\right)
\end{array}
$$

etc.

Proposition 1 Let $\mathbf{K}=1_{u}^{k}, \mathbf{L}=1_{v}^{l}$ and top $=1^{n}$. Then under the notation (9) we have the following.

1. There is a scalar $f(\mathbf{K}, \mathbf{L})$ which is nonzero for generic $u, v$ such that

$$
\check{R}^{\mathbf{L}, \mathbf{K}} \check{R}^{\mathbf{K}, \mathbf{L}}=f(\mathbf{K}, \mathbf{L}) \cdot i d
$$

2. We have

$$
\check{R}^{\mathbf{K}, t o p_{v}}=g\left(\mathbf{K}, t o p_{v}\right) \cdot P, \quad \check{R}^{t o p_{u}, \mathbf{L}}=g\left(t o p_{u}, \mathbf{L}\right) \cdot P
$$

where $P$ is the permutation and $g\left(\mathbf{K}\right.$, top $\left._{v}\right), g\left(\right.$ top $\left._{u}, \mathbf{L}\right)$ are scalars which are nonzero for generic $u, v$. 
3. The crossing symmetry holds:

$$
\begin{aligned}
\left(\check{R}^{\mathbf{K}, \mathbf{L}}\right)_{J^{\prime} I^{\prime}}^{I J} & =\left(\check{R}^{\mathbf{L}, \mathbf{K}^{*}}\right)_{I J^{\prime}}^{J I^{\prime}} \cdot \frac{f(\mathbf{K}, \mathbf{L})}{g\left(\mathbf{L}, \text { top }_{u}\right)} \\
& =\left(\check{R}^{\mathbf{L}, \mathbf{K}^{*}}\right)_{I J^{\prime}}^{J I^{\prime}} \cdot \frac{g\left(\text { top }_{u}, \mathbf{L}\right)}{f\left(\mathbf{L}, \mathbf{K}^{*}\right)}
\end{aligned}
$$
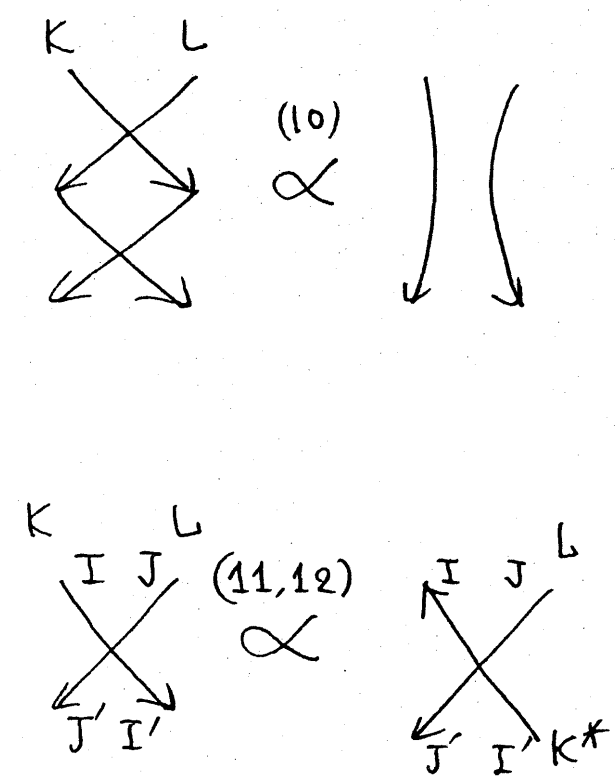

Proof. 1) follows from the first inversion formula $\check{R}^{\square_{v}, \square_{u}} \check{R}^{\square_{u}, \square_{v}}=$ scalar for the original Belavin's R-matrix. To show 2), note that the operator $\pi_{\mathbf{K}}$ commutes with the $k$-fold tensor product representation of the Heisenberg group $\langle g, h\rangle(2)$. Since $\operatorname{dim} V\left(t o p_{u}\right)=1$, the representation restricted on $V\left(t o p_{u}\right)$ is only by scalar multiplication. Together with the characterization (2) of $\check{R}^{\square_{u}, \square_{v}}$, this implies that $(1 \otimes x) \check{R}^{\mathbf{K}, \text { top }_{v}}\left(x^{-1} \otimes 1\right)=\check{R}^{\mathbf{K}, t o p_{v}}$ for any $x \in \operatorname{GL}(n)$, as desired. The fact that $g\left(\mathbf{K}, t o p_{v}\right) \neq 0$ in generic follows from the explicit calculation. To prove 3), we use 1) and an elementary braid manipulation to get

$$
\check{R}^{\mathbf{L}, \text { top }_{u}}\left(1 \otimes \pi_{\text {top }_{u}}\right)\left(\check{R}^{\mathbf{K}, \mathbf{L}} \otimes 1\right)=\pi_{\text {topu }_{u}}\left(1 \otimes \check{R}^{\mathbf{L}, \mathbf{K}^{*}}\right) \cdot f(\mathbf{K}, \mathbf{L}) .
$$



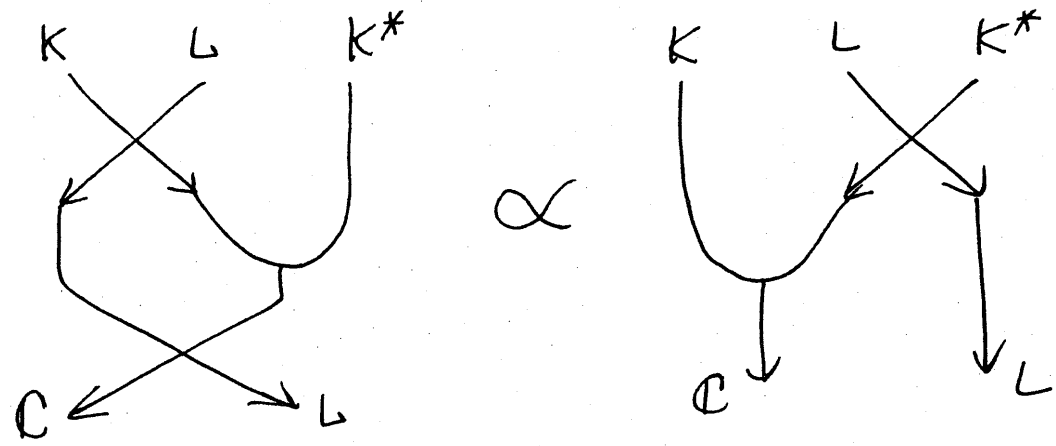

Rewriting this in terms of matrix elements we obtain (11). To get (12) is similar.

\section{Corollary 1}

$$
\frac{f(\mathbf{K}, \mathbf{L})}{g\left(\mathbf{L}, \text { top }_{u}\right)}=\frac{g\left(\text { top }_{u}, \mathbf{L}\right)}{f\left(\mathbf{L}, \mathbf{K}^{*}\right)}
$$

\section{Corollary 2}

$$
\left(\check{R}^{\mathbf{K}, \mathbf{L}}\right)_{J^{\prime} I^{\prime}}^{I J}=\left(\check{R}^{\mathbf{K}^{*}, \mathbf{L}^{*}}\right)_{J I}^{I^{\prime} J^{\prime}} \cdot \frac{f(\mathbf{K}, \mathbf{L})}{g\left(\mathbf{L}, t_{o p}\right)} \frac{f\left(\mathbf{L}, \mathbf{K}^{*}\right)}{g\left(\mathbf{K}^{*}, t_{o p}\right)} .
$$

$$
\text { K }
$$
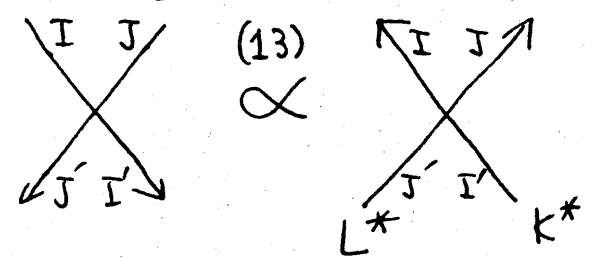

Remark. For $\mathbf{K}=1_{u}^{k}$ write $\mathbf{K}^{*^{\prime}}:=1_{u+(k-n) \hbar}^{n-k}$. We can also define a pairing by using $\check{R}^{\mathbf{K}^{*^{\prime}}, \mathbf{K}}$,

$$
<,>^{\prime}: V\left(\mathbf{K}^{*^{\prime}}\right) \otimes V(\mathbf{K}) \rightarrow V\left(t o p_{u+(k-n) \hbar}\right) \rightarrow \mathbf{C}
$$

which is also non-degenarate for generic $\hbar$ so that we can identify $V(\mathbf{K})^{*}$ and $V\left(\mathbf{K}^{*^{\prime}}\right)$. 


\section{Crossing symmetry in the face models}

As in the vertex case we can similarly derive the crossing symmetry for face models. Put

$$
\begin{aligned}
& \check{W}^{\square_{u_{1}} \square_{u_{2}} \cdots \square_{u_{l}}, \square_{v}}:=\left(\check{W}^{\square_{u_{1}}, \square_{v}}\right)^{1,2}\left(\check{W}^{\square_{u_{2}}, \square_{v}}\right)^{2,3} \cdots\left(\check{W}^{\square_{u_{l}}, \square_{v}}\right)^{l, l+1},
\end{aligned}
$$

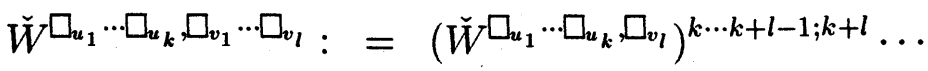

$$
\begin{aligned}
& \left(\check{W}^{\square_{u_{1}} \cdots \square_{u_{k}}, \square_{v_{2}}}\right)^{2 \cdots k+1 ; k+2}\left(\check{W}^{\square_{u_{1}} \cdots \square_{u_{k}}, \square_{v_{1}}}\right)^{1 \cdots k ; k+1},
\end{aligned}
$$

where the superscripts denote the components they act on. The fusion operator for the face model [JKMO] associated with $1^{k}$ is given by

$$
\begin{gathered}
\Pi_{1^{k}}:=\left(\check{W}^{\square_{u_{1}}, \square_{u_{2}}}\right)^{k-1 ; k} \cdots\left(\check{W}^{\square_{u_{1}} \square_{u_{2}} \cdots \square_{u_{k-2}}, \square_{u_{k-1}}}\right)^{2 \cdots k-1 ; k}\left(\check{W}^{\square_{u_{1}} \square_{u_{2}} \cdots \square_{u_{k-1}}, \square_{u_{k}}}\right)^{1 \cdots k-1 ; k} \\
: \mathcal{P}_{\square_{u} \cdots \square_{u+(k-1) \hbar}} \rightarrow \mathcal{P}_{\square_{u+(k-1) \hbar} \cdots \square_{u}}
\end{gathered}
$$

where the spectral parameters are specialized as before $(7):\left(u_{1}, \cdots, u_{k}\right)=$ $(u, \cdots, u+(k-1) \hbar)$.

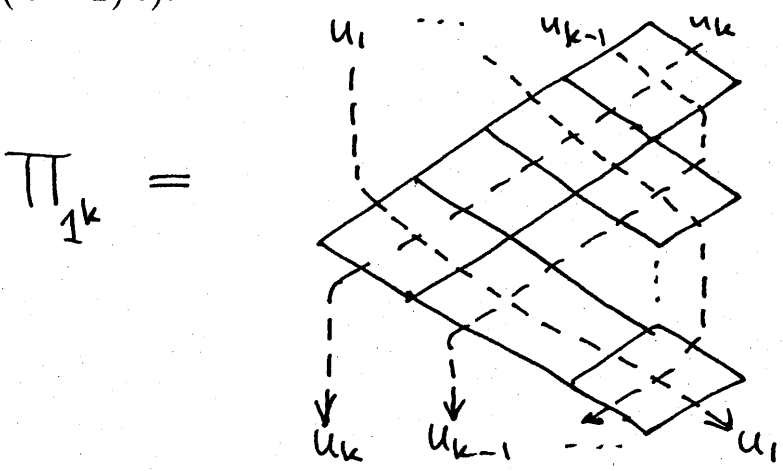

We denote the image of $\Pi_{1^{k}}$ in $\mathcal{P}_{\square_{u+(k-1) \hbar} \cdots \square_{u}}$ (resp. $\mathcal{P}_{\lambda \square_{u+(k-1) \hbar} \cdots \square_{u}}$ ) as $\mathcal{P}_{1_{u}^{k}}$ (resp. $\mathcal{P}_{\lambda 1_{u}^{k}}^{\nu}$ ), or $\mathcal{P}_{\mathbf{K}}$ (resp. $\mathcal{P}_{\lambda \mathbf{K}}^{\nu}$ ) with the shorthand in the previous section $\mathbf{K}=1_{u}^{k}$. We also write $\mathcal{P}_{\lambda \mathbf{K L}}^{\nu}:=\oplus_{\mu} \mathcal{P}_{\lambda \mathbf{K}}^{\mu} \otimes \mathcal{P}_{\mu \mathbf{L}}^{\nu}, \mathcal{P}_{\mathbf{K L}}:=\oplus_{\lambda \nu} \mathcal{P}_{\lambda \mathbf{K L}}^{\nu}$. It turns out that for $\mathbf{K}=1_{u}^{k}$ and generic value of $\hbar$ the dimension of the space $\mathcal{P}_{\lambda \mathbf{K}}^{\nu}$ is 
given by

$$
\operatorname{dim} \mathcal{P}_{\lambda \mathbf{K}}^{\nu}=\left|\left\{\left(j_{1}, \cdots, j_{k}\right) ; 1 \leq j_{1}<\cdots<j_{k} \leq n, \overline{{j_{1}}_{1}}+\cdots+\overline{{j_{k}}}=\nu-\lambda\right\}\right|
$$

which is equal to the multiplicity of the weight $\nu-\lambda$ of the $G L\left(\mathbf{C}^{n}\right)$-module $\wedge^{k}\left(\mathbf{C}^{n}\right)$. In particular, for top $=1^{n}$ we have $\operatorname{dim} \mathcal{P}_{\lambda t o p_{u}}^{\nu}=\delta_{\lambda, \nu}$.

The fused weight for $\mathbf{K}=1_{u}^{k}, \mathbf{L}=1_{v}^{l}$ is defined by

$$
\check{W}^{\mathbf{K}, \mathbf{L}}:=\left.\check{W}^{\square_{u+(k-1) \hbar} \cdots \square_{u}, \square_{v+(l-1) \hbar} \cdots \square_{v}}\right|_{\mathcal{P}_{\mathbf{K L}}}: \mathcal{P}_{\mathbf{K L}} \rightarrow \mathcal{P}_{\mathbf{L K}}
$$

and they satisfy

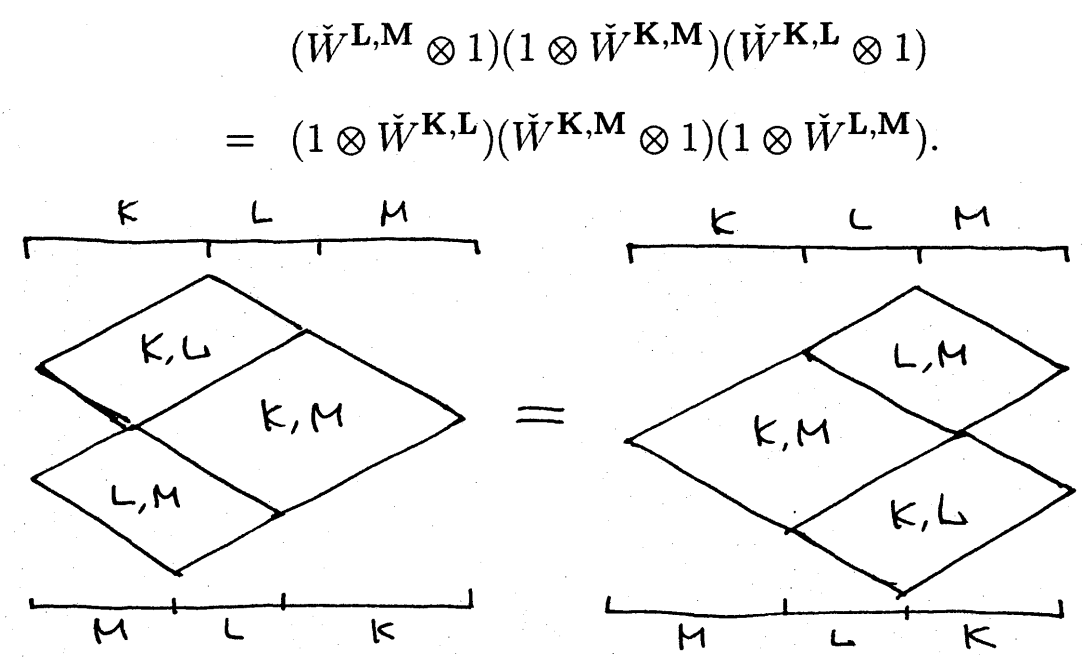

Fix a base $\mid t o p_{\lambda, u}>\in \mathcal{P}_{\lambda \text { topu }_{u}}^{\lambda}$ for each $\lambda$ and $u$. We can define the pairing

$$
<,>:\left(\mathcal{P}_{\mathbf{K}}\right)^{*} \otimes \mathcal{P}_{\mathbf{K}^{*}} \rightarrow \mathcal{P}_{\text {top }_{u}} \rightarrow \mathbf{C}
$$

as the composition of $\check{W}^{\mathbf{K}, \mathbf{K}^{*}}$ and the identification map $\mid t_{0 p_{\lambda, u}}>\mapsto 1$. Suppose $\mathcal{P}_{\lambda \mathbf{K}}^{\mu} \neq 0$. Then for generic $\hbar$ this pairing is non-degenarate between $\left(\mathcal{P}_{\lambda \mathbf{K}}^{\mu}\right)^{*}$ and $\mathcal{P}_{\mu \mathrm{K}^{*}}^{\lambda}$ so that we can identify these spaces with each other. Take basis $\left\{e_{\lambda a}^{\mu}\right\}_{a}$ of $\mathcal{P}_{\lambda \mathbf{K}}^{\mu}$ and its dual basis (with respect to $\left.<,>\right)\left\{e_{\mu a}^{* \lambda}\right\}_{a}$ of $\mathcal{P}_{\mu \mathbf{K}^{*}}^{\lambda}$ and define the matrix element of $\check{W}$ as follows.

$$
\check{W}^{\mathbf{K}, \mathbf{L}}\left(e_{\lambda a}^{\mu} \otimes e_{\mu b}^{\nu}\right)=\sum_{b^{\prime} \mu^{\prime} a^{\prime}} e_{\lambda b^{\prime}}^{\mu^{\prime}} \otimes e_{\mu^{\prime} a^{\prime}}^{\nu} \cdot \check{W}^{\mathbf{K}, \mathbf{L}}\left[\begin{array}{ccc}
a & \mu & b \\
\lambda & & \nu \\
b^{\prime} & \mu^{\prime} & a^{\prime}
\end{array}\right]
$$




$$
\left(e_{\lambda a}^{\mu} \in \mathcal{P}_{\lambda \mathbf{K}}^{\mu}, e_{\mu b}^{\nu} \in \mathcal{P}_{\mu \mathbf{L}}^{\nu}\right)
$$
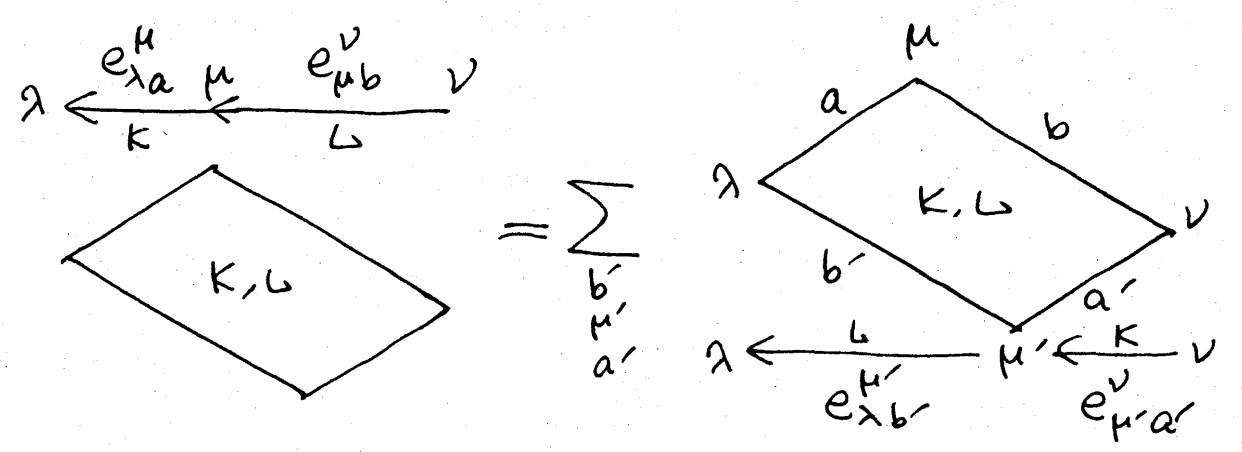

Proposition $\mathbf{2}$ Let $\mathbf{K}, \mathbf{L}$ are as in Propositon 1 and let $f(\mathbf{K}, \mathbf{L})$ be the scalar therein (10).

1. We have

$$
\check{W}^{\mathbf{L}, \mathbf{K}} \check{W}^{\mathbf{K}, \mathbf{L}}=f(\mathbf{K}, \mathbf{L}) \cdot i d
$$

2. For each $\lambda$ and $\mu$ there exists a scalar $G_{\lambda}^{\mu}\left(\mathbf{K}\right.$, top $\left._{v}\right)\left(\right.$ resp. $G_{\lambda}^{\mu}\left(\right.$ top $\left.\left._{u}, \mathbf{L}\right)\right)$ which is non zero for generic $u, v$ and satisfies that

$$
\begin{gathered}
\check{W}^{\mathbf{K}, \text { top }_{v}}\left(b \otimes \mid \text { top }_{\mu, v}>\right)=\mid \operatorname{top}_{\lambda, v}>\otimes b \cdot G_{\lambda}^{\mu}\left(\mathbf{K}, \text { top }_{v}\right) \quad \text { for any } b \in \mathcal{P}_{\lambda \mathbf{K}}^{\mu} \\
\left(\text { resp. } \check{W}^{\mathbf{L}}\left(\mid \operatorname{top}_{\lambda, u}>\otimes b\right)=\left(b \otimes \mid \text { top }_{\mu, u}>\right) \cdot G_{\lambda}^{\mu}(\text { top }, \mathbf{L})\right. \text { for any } \\
\left.b \in \mathcal{P}_{\lambda t o p_{u}, \mathbf{L}}^{\mu}\right) .
\end{gathered}
$$

3. The crossing symmetry:

$$
\begin{aligned}
\check{W}^{\mathbf{K}, \mathbf{L}}\left[\begin{array}{ccc}
a & \mu & b \\
\lambda & & \nu \\
b^{\prime} & \mu^{\prime} & a^{\prime}
\end{array}\right] & =\check{W}^{\mathbf{L}, \mathbf{K}^{*}}\left[\begin{array}{ccc}
b & \nu & a^{\prime} \\
\mu & & \mu^{\prime} \\
a & \lambda & b^{\prime}
\end{array}\right] \cdot \frac{f(\mathbf{K}, \mathbf{L})}{G_{\lambda}^{\mu^{\prime}}\left(\mathbf{L}, t o p_{u}\right)} \\
& =\check{W}^{\mathbf{L}, \mathbf{K}^{*}}\left[\begin{array}{ccc}
b & \nu & a^{\prime} \\
\mu & & \mu^{\prime} \\
a & \lambda & b^{\prime}
\end{array}\right] \cdot \frac{G_{\lambda}^{\mu^{\prime}}\left(t o p_{u}, \mathbf{L}\right)}{f\left(\mathbf{L}, \mathbf{K}^{*}\right)}
\end{aligned}
$$



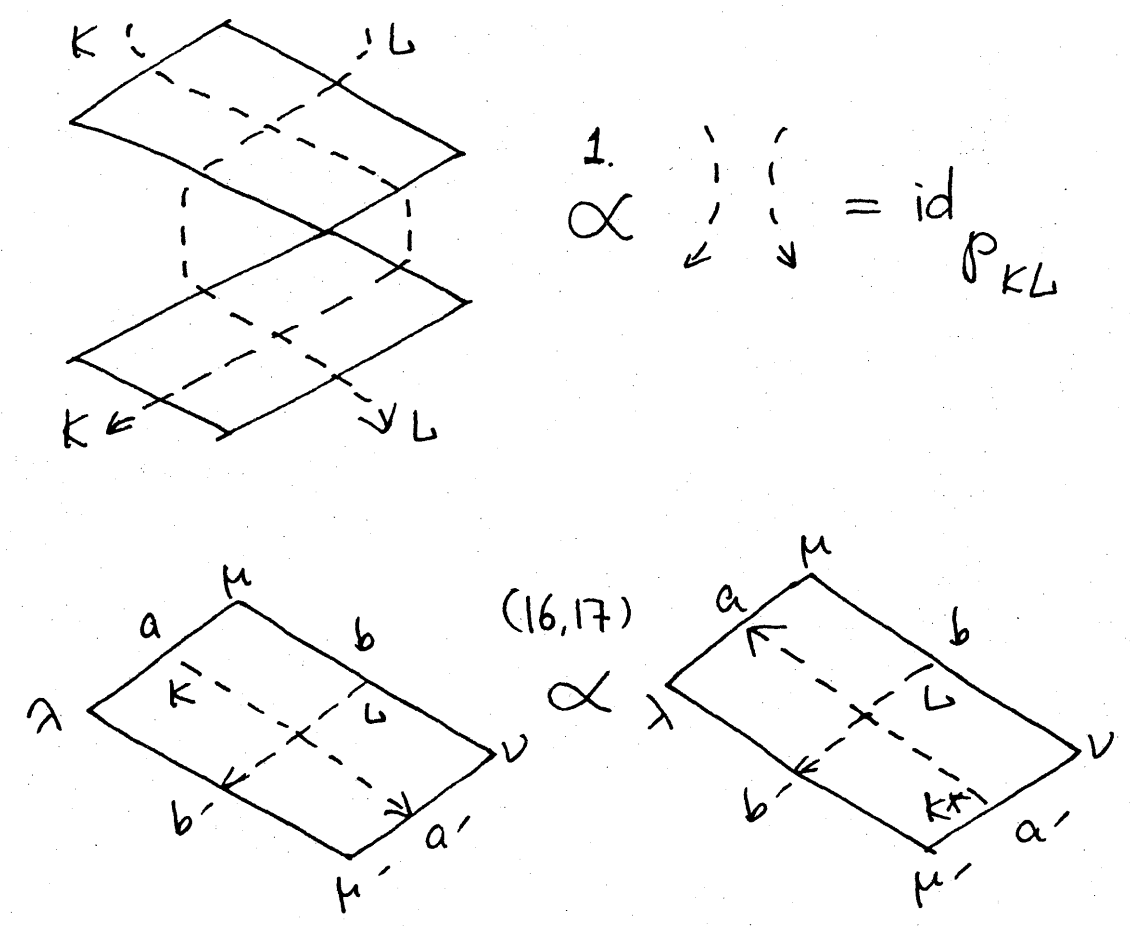

Proof. Proof of 1) is similar to the vertex case. 2) is trivial when $k=1$ because the space $\mathcal{P}_{\lambda \square_{u}}^{\mu}$ is at most one dimensional, and then the general case follows. The fact that $G_{\lambda}^{\mu}\left(\mathbf{K}, t o p_{v}\right) \neq 0$ in generic follows from calculation. To prove 3 ), as in the vertex case we use 1) and an elementary braid manipulation to get

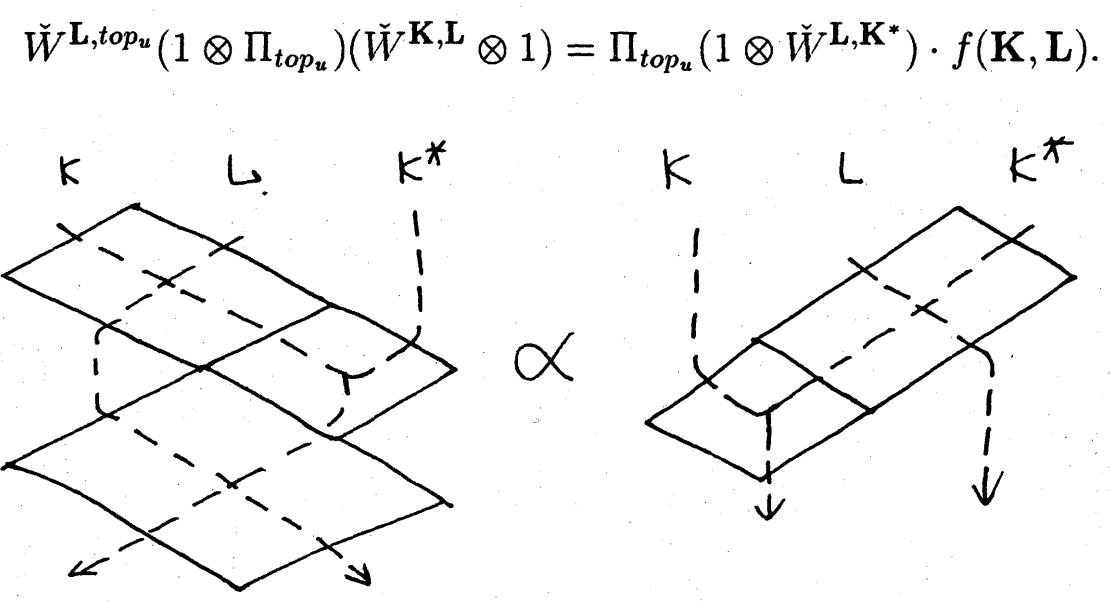

Rewriting this in terms of matrix elements we obtain (16). Similarly we get (17). 
Corollary 3

$$
\frac{f(\mathbf{K}, \mathbf{L})}{G_{\lambda}^{\mu}\left(\mathbf{L}, t_{o p}\right)}=\frac{G_{\lambda}^{\mu}\left(t o p_{u}, \mathbf{L}\right)}{f\left(\mathbf{L}, \mathbf{K}^{*}\right)}
$$

Corollary 4

$$
\check{W}^{\mathbf{K}, \mathbf{L}}\left[\begin{array}{ccc}
a & \mu & b \\
\lambda & & \nu \\
b^{\prime} & \mu^{\prime} & a^{\prime}
\end{array}\right]=\check{W}^{\mathbf{K}^{*}, \mathbf{L}^{*}}\left[\begin{array}{ccc}
a^{\prime} & \mu^{\prime} & b^{\prime} \\
\nu & & \lambda \\
b & \mu & a
\end{array}\right] \cdot \frac{f(\mathbf{K}, \mathbf{L})}{G_{\lambda}^{\mu^{\prime}\left(\mathbf{L}, t o p_{u}\right)}} \frac{f\left(\mathbf{L}, \mathbf{K}^{*}\right)}{G_{\mu}^{\lambda}\left(\mathbf{K}^{*}, t o p_{v}\right)} .
$$
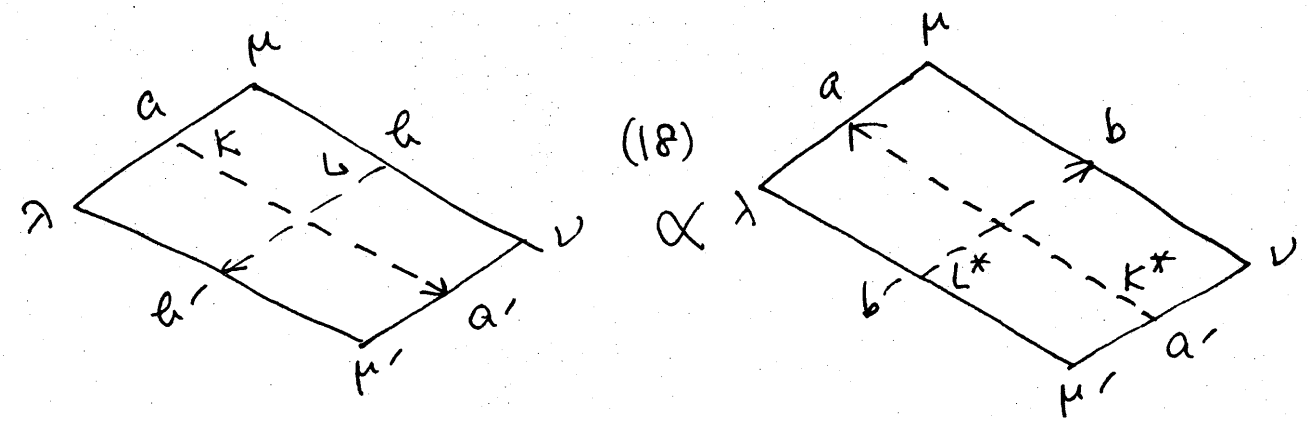

Remark. As in the vertex case we can define a non-degenerate pairing

$$
<,>^{\prime}: \mathcal{P}_{\mathbf{K}^{*^{\prime}}} \otimes \mathcal{P}_{\mathbf{K}} \rightarrow \mathcal{P}_{\text {top }_{u+(k-n) \hbar}} \rightarrow \mathbf{C}
$$

by using $\check{W}^{\mathbf{K}^{*^{\prime}}, \mathbf{K}}$, where $\mathbf{K}^{*^{\prime}}$ is the same as before (14).

\section{The incoming intertwining vectors}

Fusion of the intertwining vector. Let $\mathbf{K}=1_{u}^{k}, \mathbf{L}=1_{v}^{l}$, top $=1^{n}$ as before. Let us consider the operator

$$
\begin{aligned}
\phi_{\lambda \square_{u_{1}} \square_{u_{2}} \cdots \square_{u_{k}}}^{\nu}:=\oplus_{\mu_{1}, \mu_{2} \cdots, \mu_{k-1}} \phi_{\lambda \square_{u_{1}}}^{\mu_{1}} \otimes \phi_{\mu_{1} \square_{u_{2}}}^{\mu_{2}} \otimes \cdots \otimes \phi_{\mu_{k-1} \square_{u_{k}}}^{\nu} \\
: \mathcal{P}_{\lambda \square_{u_{1}} \square_{u_{2}} \cdots \square_{u_{k}}}^{\nu}=\oplus_{\mu_{1}, \mu_{2} \cdots, \mu_{k-1}} \mathcal{P}_{\lambda \square_{u_{1}}}^{\mu_{1}} \otimes \mathcal{P}_{\mu_{1} \square_{u_{2}}}^{\mu_{2}} \otimes \cdots \otimes \mathcal{P}_{\mu_{k-1} \square_{u_{k}}}^{\nu}
\end{aligned}
$$




$$
\longrightarrow V\left(\square_{u_{1}}\right) \otimes V\left(\square_{u_{2}}\right) \otimes \cdots \otimes V\left(\square_{u_{k}}\right)
$$

then from the intertwining property (5) we have

$$
\pi_{1^{k}} \phi_{\lambda \square_{u} \square_{u+\hbar} \cdots \square_{u+(k-1) \hbar}}^{\nu}=\phi_{\lambda \square_{u+(k-1) \hbar} \cdots \square_{u+\hbar} \square_{u}}^{\nu} \Pi_{1^{k}}
$$
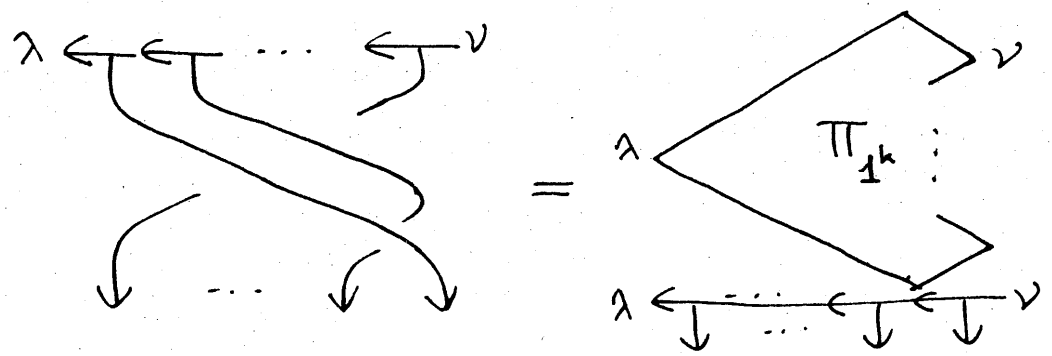

This implies that the image of the restriction $\phi_{\lambda \mathbf{K}}^{\nu}:=\left.\phi_{\lambda \square_{u+(k-1) \hbar} \cdots \square_{u+\hbar} \square_{u}}^{\nu}\right|_{\mathcal{P}_{\mathbf{K}}^{\nu}}$ lies in $V(\mathbf{K})$ :

$$
\phi_{\lambda \mathbf{K}}^{\nu}: \mathcal{P}_{\lambda \mathbf{K}}^{\nu} \rightarrow V(\mathbf{K})
$$

Generalizing the intertwining property of the original intertwining vector (5), these 'fused intertwining vectors' intertwine the fused R-matrix and the fused face operators: Let

$$
\operatorname{pr}_{\lambda \mu_{0} \nu}^{\mathbf{L}, \mathbf{K}}: \oplus_{\mu^{\prime}} \mathcal{P}_{\lambda \mathbf{L}}^{\mu^{\prime}} \otimes \mathcal{P}_{\mu^{\prime} \mathbf{K}}^{\nu} \rightarrow \mathcal{P}_{\lambda \mathbf{L}}^{\mu_{0} \cdot} \otimes \mathcal{P}_{\mu_{0} \mathbf{K}}^{\nu}
$$

denotes the projection and put

$$
\check{W}^{\mathbf{K}, \mathbf{L}}\left[\begin{array}{ccc} 
& \mu & \\
\lambda & & \nu \\
& & \\
& \mu^{\prime} &
\end{array}\right]:=\left.\operatorname{pr}_{\lambda \mu^{\prime} \nu}^{\mathbf{L}, \mathbf{K}} \cdot \check{W}^{\mathbf{K}, \mathbf{L}}\right|_{\mathcal{P}_{\lambda \mathbf{K}}^{\mu}{ }_{\mu}{ }_{\mu} \mathbf{L}}: \mathcal{P}_{\lambda \mathbf{K}}^{\mu} \otimes \mathcal{P}_{\mu \mathbf{L}}^{\nu} \rightarrow \mathcal{P}_{\lambda \mathbf{L}}^{\mu^{\prime}} \otimes \mathcal{P}_{\mu^{\prime} \mathbf{K}}^{\nu}
$$

Then it is easy to see that

$$
\check{R}^{\mathbf{K}, \mathbf{L}} \phi_{\lambda \mathbf{K}}^{\mu} \otimes \phi_{\mu \mathbf{L}}^{\nu}=\sum_{\mu^{\prime}} \phi_{\lambda \mathbf{L}}^{\mu^{\prime}} \otimes \phi_{\mu^{\prime} \mathbf{K}}^{\nu} \check{W}^{\mathbf{K}, \mathbf{L}}\left[\begin{array}{ccc} 
& \mu & \\
& & \\
\lambda & & \nu \\
& & \mu^{\prime}
\end{array}\right]
$$


where the both hand sides are the operators $\mathcal{P}_{\lambda \mathbf{K}}^{\mu} \otimes \mathcal{P}_{\mu \mathbf{L}}^{\nu} \rightarrow V(\mathbf{L}) \otimes V(\mathbf{K})$.
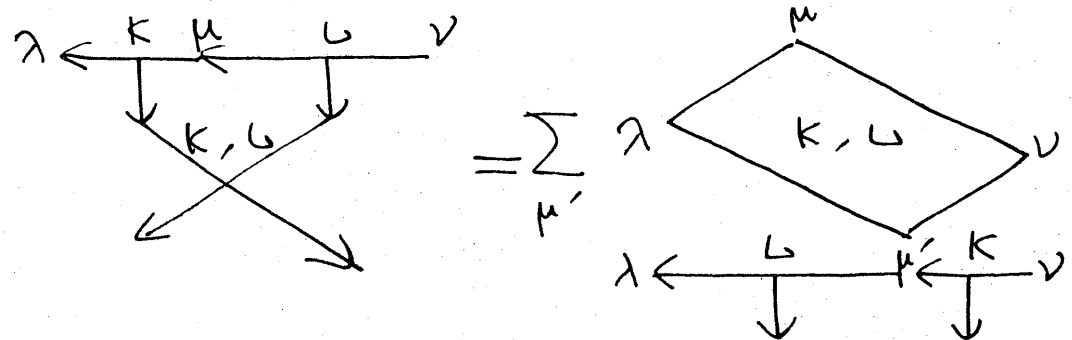

The incoming intertwining vectors. While the fused intertwining vectors (19) may be called outgoing intertwining vectors because the space $V(\mathbf{K})$ appears there as the output of these quantities. In contrast with this, what we would like to call 'incoming' intertwining vectors are the quantities

$$
\phi_{\lambda}^{\mu \mathbf{L}}: V(\mathbf{L}) \rightarrow \mathcal{P}_{\lambda}^{\mu \mathbf{L}}
$$

that satisfy

$$
\phi_{\lambda}^{\mu \mathbf{L}} \otimes \phi_{\mu}^{\nu \mathbf{K}} \check{R}^{\mathbf{K}, \mathbf{L}}=\sum_{\mu^{\prime}} \check{W}^{\mathbf{K}, \mathbf{L}}\left[\begin{array}{ccc} 
& \mu^{\prime} & \\
\lambda & & \nu \\
& &
\end{array}\right] \phi_{\lambda}^{\mu^{\prime} \mathbf{K}} \otimes \phi_{\mu^{\prime}}^{\nu \mathbf{L}}
$$
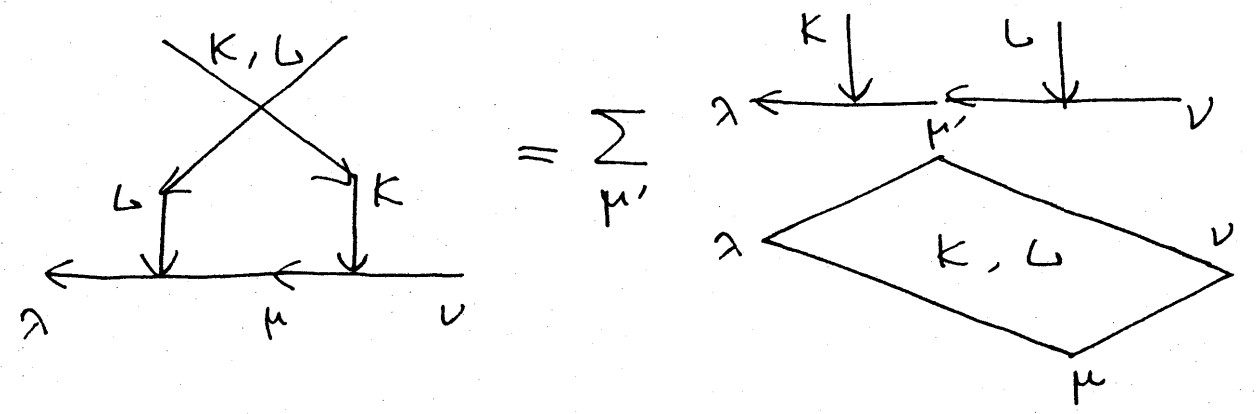

and now we are in the position to construct them. 
First we substitute $k$ by $n-k$ and $l$ by $n-l$ in (20),

$$
\check{R}^{\mathbf{K}^{*}, \mathbf{L}^{*}} \phi_{\nu \mathbf{K}^{*}}^{\mu} \otimes \phi_{\mu \mathbf{L}^{*}}^{\lambda}=\sum_{\mu^{\prime}} \phi_{\nu \mathbf{L}^{*}}^{\mu^{\prime}} \otimes \phi_{\mu^{\prime} \mathbf{K}}^{\lambda} \check{W}^{\mathbf{K}^{*}, \mathbf{L}^{*}}\left[\begin{array}{ccc} 
& \mu & \\
& & \lambda \\
\nu & & \lambda \\
& \mu^{\prime} &
\end{array}\right]
$$

and take the matrix elements: write

$$
\phi_{\lambda \mathbf{K}}^{\nu}\left(e_{\lambda a}^{\nu}\right)=\sum_{I} e^{I}\left(\phi_{\lambda \mathbf{K}}^{\nu}\right)_{I, a} \in V(\mathbf{K})
$$

then we have

$$
\begin{gathered}
\sum_{I J}\left(\check{R}^{\mathbf{K}^{*}, \mathbf{L}^{*}}\right)_{J^{\prime} I^{\prime}}^{I J}\left(\phi_{\nu \mathbf{K}}^{\mu}\right)_{I a}\left(\phi_{\mu \mathbf{L}^{*}}^{\lambda}\right)_{J b} \\
=\sum_{\mu^{\prime} a^{\prime} b^{\prime}}\left(\phi_{\nu \mathbf{L}^{*}}^{\mu^{\prime}}\right)_{J^{\prime} b^{\prime}}\left(\phi_{\mu^{\prime} \mathbf{K}^{*}}^{\lambda}\right)_{I^{\prime} a^{\prime}} \check{W}^{\mathbf{K}^{*} \mathbf{L}^{*}}\left[\begin{array}{ccc}
a & \mu & b \\
\nu & & \lambda \\
b^{\prime} & \mu^{\prime} & a^{\prime}
\end{array}\right] .
\end{gathered}
$$

We use the crossing symmetries (13), (18) in Corollaries 2, 4 to get:

$$
\begin{gathered}
\sum_{I J}\left(\check{R}^{\mathbf{K}, \mathbf{L}}\right)_{J I}^{I^{\prime} J^{\prime}} \frac{g\left(\mathbf{L}, t o p_{u}\right)}{f(\mathbf{K}, \mathbf{L})} \frac{g\left(\mathbf{K}^{*}, t o p_{v}\right)}{f\left(\mathbf{L}, \mathbf{K}^{*}\right)} \cdot\left(\phi_{\nu \mathbf{K}^{*}}^{\mu}\right)_{I a}\left(\phi_{\mu \mathbf{L}^{*}}^{\lambda}\right)_{J b} \\
=\sum_{\mu^{\prime} a^{\prime} b^{\prime}}\left(\phi_{\nu \mathbf{L}^{*}}^{\mu^{\prime}}\right)_{J^{\prime} b^{\prime}}\left(\phi_{\mu^{\prime} \mathbf{K}^{*}}^{\lambda}\right)_{I^{\prime} a^{\prime}} \check{W}^{\mathbf{K}, \mathbf{L}}\left[\begin{array}{rrr}
a^{\prime} & \mu^{\prime} & b^{\prime} \\
\lambda & & \nu \\
b & \mu & a
\end{array}\right] \cdot \frac{G_{\lambda}^{\mu}\left(\mathbf{L}, t o p_{u}\right)}{f(\mathbf{K}, \mathbf{L})} \frac{G_{\mu^{\prime}}^{\lambda}\left(\mathbf{K}^{*}, t o p_{v}\right)}{f\left(\mathbf{L}, \mathbf{K}^{*}\right)}
\end{gathered}
$$

We need the following.

Lemma 1 Let $<\phi_{\mu t o p_{v}}^{\mu}>\in \mathbf{C}$ be the coefficient in the formula

$$
\phi_{\mu t o p_{v}}^{\mu} e_{\mu t o p_{v}}^{\mu}=e_{t o p_{v}}<\phi_{\mu t o p_{v}}^{\mu}>
$$

where $e_{\mu t o p_{v}}^{\mu} \in \mathcal{P}_{\mu t o p_{v}}^{\mu}$ (resp. $e_{\text {top }} \in V\left(\right.$ top $\left._{v}\right)$ ) denotes a fixed basis of the one dimensional space. Then we have the formula

$$
\frac{G_{\lambda}^{\mu}\left(\mathbf{K}, \text { top }_{v}\right)}{g\left(\mathbf{K}, \text { top }_{v}\right)}=\frac{<\phi_{\mu t o p_{v}}^{\mu}>}{\left\langle\phi_{\lambda t o p_{v}}^{\lambda}\right\rangle} .
$$


Proof. Recall the formula (20) for $l=n$,

$$
\check{R}^{\mathbf{K}, \text { top }_{v}} \phi_{\lambda \mathbf{K}}^{\mu} \otimes \phi_{\mu t o p_{v}}^{\mu}=\sum_{\mu^{\prime}} \phi_{\lambda t o p_{v}}^{\mu^{\prime}} \otimes \phi_{\mu^{\prime} \mathbf{K}}^{\mu} \check{W}^{\mathbf{K} t o p_{v}}\left[\begin{array}{ccc} 
& \mu \\
\lambda & & \mu \\
& & \mu^{\prime}
\end{array}\right]
$$

Since $\operatorname{dim} \mathcal{P}_{\lambda t o p_{u}}^{\mu}=\delta_{\lambda \mu}$, the summand in the right hand side is zero unless $\mu^{\prime}=\lambda$. Then the lemma follows if we take the matrix elements of the both hand sides with using Proposition 2(2).

Applying (23) to (22) we have:

$$
\begin{aligned}
& \sum_{I J}\left(\check{R}^{\mathbf{K}, \mathbf{L}}\right)_{J I}^{I^{\prime} J^{\prime}} \cdot\left(\phi_{\nu \mathbf{K}}^{\mu}\right)_{I a}\left(\phi_{\mu \mathbf{L}^{*}}^{\lambda}\right)_{J b} \\
= & \sum_{\mu^{\prime} a^{\prime} b^{\prime}}\left(\phi_{\nu \mathbf{L}^{*}}^{\mu^{\prime}}\right)_{J^{\prime} b^{\prime}}\left(\phi_{\mu^{\prime} \mathbf{K}^{*}}^{\lambda}\right)_{I^{\prime} a^{\prime}} \check{W}^{\mathbf{K}, \mathbf{L}}\left[\begin{array}{lll}
a^{\prime} & \mu^{\prime} & b^{\prime} \\
\lambda & & \nu \\
b & \mu & a
\end{array}\right] \cdot \frac{G_{\lambda}^{\mu}\left(\mathbf{L}, t o p_{u}\right)}{g\left(\mathbf{L}, t o p_{u}\right)} \frac{G_{\mu^{\prime}}^{\lambda}\left(\mathbf{K}^{*}, t o p_{v}\right)}{g\left(\mathbf{K}^{*}, t o p_{v}\right)} \\
= & \sum_{\mu^{\prime} a^{\prime} b^{\prime}}\left(\phi_{\nu \mathbf{L}^{*}}^{\mu^{\prime}}\right)_{J^{\prime} b^{\prime}}\left(\phi_{\mu^{\prime} \mathbf{K}^{*}}^{\lambda}\right)_{I^{\prime} a^{\prime}} \check{W}^{\mathbf{K}, \mathbf{L}}\left[\begin{array}{lll}
a^{\prime} & \mu^{\prime} & b^{\prime} \\
\lambda & & \nu \\
b & \mu & a
\end{array}\right] \cdot \frac{\left\langle\phi_{\mu t o p_{u}}^{\mu}>\left\langle\phi_{\lambda t o p_{v}}^{\lambda}>\right.\right.}{<\phi_{\lambda t o p_{u}}^{\lambda}><\phi_{\mu^{\prime} t o p_{v}}^{\mu^{\prime}}>} .
\end{aligned}
$$

Deviding the both hand sides by $<\phi_{\mu t o p_{u}}^{\mu}><\phi_{\lambda t o p_{v}}^{\lambda}>$ we get

$\sum_{I J}\left(\check{R}^{\mathbf{K}, \mathbf{L}}\right)_{J I}^{I^{\prime} J^{\prime}} \frac{\left(\phi_{\nu \mathbf{K}^{*}}^{\mu}\right)_{I a}}{\left\langle\phi_{\mu t o p_{u}}^{\mu}\right\rangle} \frac{\left(\phi_{\mu \mathbf{L}^{*}}^{\lambda}\right)_{J b}}{\left\langle\phi_{\lambda t o p_{v}}^{\lambda}\right\rangle}=\sum_{\mu^{\prime} a^{\prime} b^{\prime}} \frac{\left(\phi_{\nu \mathbf{L}^{*}}^{\mu^{\prime}}\right)_{J^{\prime} b^{\prime}}}{\left\langle\phi_{\mu^{\prime} t o p_{v}}^{\mu^{\prime}}>\right.} \frac{\left(\phi_{\mu^{\prime} \mathbf{K}^{*}}^{\lambda}\right)_{I^{\prime} a^{\prime}}}{<\phi_{\lambda t o p_{u}}^{\lambda}>} \check{W}^{\mathbf{K}, \mathbf{L}}\left[\begin{array}{ccc}a^{\prime} & \mu^{\prime} & b^{\prime} \\ \lambda & \nu \\ b & \mu & a\end{array}\right]$

Thus we obtained the desired incoming intertwining vectors (21):

Theorem 1 For each $\lambda, \nu$ and $\mathbf{K}=1_{u}^{k}$ define the operator

$$
\phi_{\lambda}^{\nu \mathbf{K}}: V(\mathbf{K}) \rightarrow \mathcal{P}_{\lambda \mathbf{K}}^{\nu}
$$

by

$$
\phi_{\lambda}^{\nu \mathrm{K}}\left(e^{I}\right):=\sum_{a} e_{\lambda a}^{\nu} \frac{\left(\phi_{\nu \mathrm{K}^{*}}^{\lambda}\right)_{I a}}{\left\langle\phi_{\lambda t o p_{u}}^{\lambda}\right\rangle}
$$


for each basis element $e^{I} \in V(\mathbf{K})$, where $\mathbf{K}^{*}:=1_{u+k \hbar}^{n-k}$ (9).

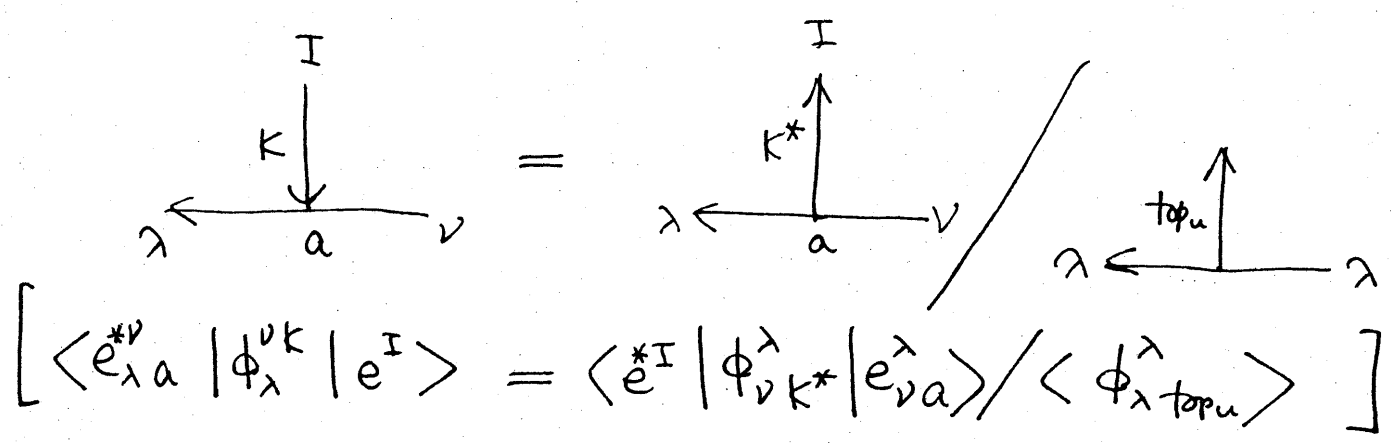

Then they satisfy

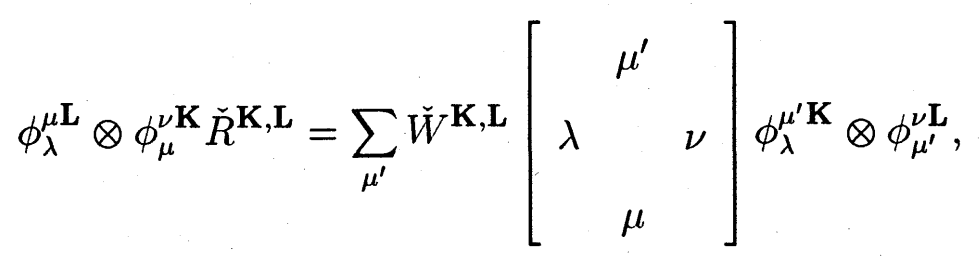

where the both hand sides are the operators $V(\mathbf{K}) \otimes V(\mathbf{L}) \rightarrow \mathcal{P}_{\lambda \mathbf{L}}^{\mu} \otimes \mathcal{P}_{\mu \mathbf{K}}^{\nu}$.

By the construction incoming vectors and outgoing ones satisfy the following duality relations [BKMS].

$$
\begin{aligned}
& \sum_{\lambda} \phi_{\lambda \mathbf{K}}^{\mu} \phi_{\lambda}^{\mu \mathbf{K}}=i d_{V(\mathbf{K})}: V(\mathbf{K}) \rightarrow \mathcal{P}_{\mathbf{K}} \rightarrow V(\mathbf{K}), \\
& \phi_{\lambda}^{\mu \mathbf{K}} \phi_{\nu \mathbf{K}}^{\mu}=\delta_{\lambda, \nu} i d_{\mathcal{P}_{\lambda \mathbf{K}}^{\mu}}: \mathcal{P}_{\nu \mathbf{K}}^{\mu} \rightarrow V(\mathbf{K}) \rightarrow \mathcal{P}_{\lambda \mathbf{K}}^{\mu} .
\end{aligned}
$$

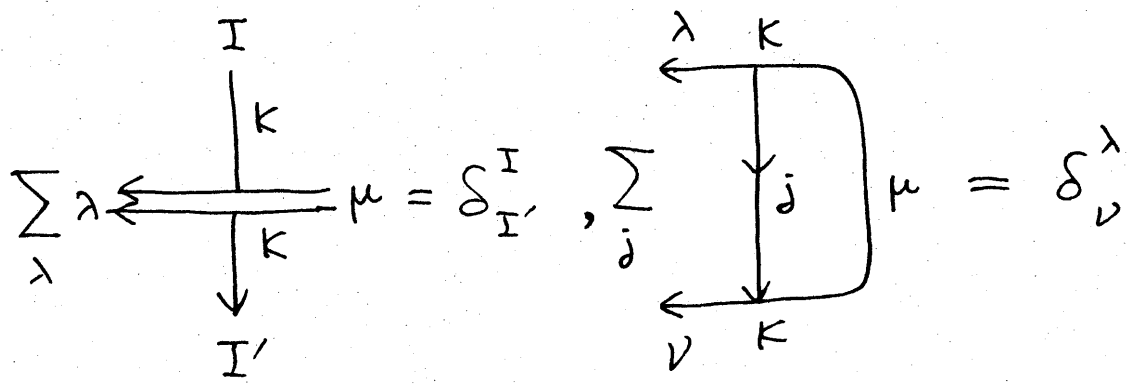




\section{The L-operator}

We define the vector space

$$
\mathcal{V}:=\Pi_{\mu \in \mathbf{h}^{*}} \mathbf{C} \delta^{\mu}
$$

with $\delta^{\mu}$, the "delta function supported at $\mu \in \mathbf{h}^{*}$ ", as its basis.

Theorem 2 For each $\lambda, \mu \in \mathbf{h}^{*}$ put

$$
\check{L}(\mathbf{K})_{\lambda}^{\mu}:=\phi_{\lambda \mathbf{K}}^{\mu} \phi_{\lambda}^{\mu \mathbf{K}}: V(\mathbf{K}) \rightarrow \mathcal{P}_{\lambda \mathbf{K}}^{\mu} \rightarrow V(\mathbf{K})
$$

for $\mathbf{K}=1_{u}^{k}$ and define the operator

$$
\check{L}(\mathbf{K}): V(\mathbf{K}) \otimes \mathcal{V} \rightarrow \mathcal{V} \otimes V(\mathbf{K})
$$

by

$$
\check{L}(\mathbf{K})\left(\mathrm{v} \otimes \delta^{\mu}\right):=\sum_{\lambda} \delta^{\lambda} \otimes \check{L}(\mathbf{K})_{\lambda}^{\mu}(\mathrm{v})
$$

for any $\mathrm{v} \in V(\mathbf{K})$ and $\mu \in \mathbf{h}^{*}$. Then this operator is well defined and satisfies the following:

$$
\begin{aligned}
& (\check{L}(\mathbf{L}) \otimes 1)(1 \otimes \check{L}(\mathbf{K}))\left(\check{R}^{\mathbf{K}, \mathbf{L}} \otimes 1\right) \\
= & \left(1 \otimes \check{R}^{\mathbf{K}, \mathbf{L}}\right)(\check{L}(\mathbf{K}) \otimes 1)(1 \otimes \check{L}(\mathbf{L}))
\end{aligned}
$$

where $\mathbf{K}=1_{u}^{k}, \mathbf{L}=1_{v}^{l}$ and both hand sides are operators

$$
V(\mathbf{K}) \otimes V(\mathbf{L}) \otimes \mathcal{V} \rightarrow \mathcal{V} \otimes V(\mathbf{L}) \otimes V(\mathbf{K})
$$

In particular, putting $k=l=1$ the operator $\check{L}\left(\square_{v}\right)$ gives an L-operator for Belavin's R-matrix $\check{R}^{\square_{u}}, \square_{v}$. 


$$
L^{v}(k)_{\lambda}^{\mu}=\lambda \Leftarrow \frac{\downarrow^{k}}{\downarrow^{k}} \mu
$$

Proof. Remark that for each $\lambda, \check{L}(\mathbf{K})_{\mu}^{\lambda}=0$ for all but finite $\mu$, which imply that the operator $\check{L}(\mathbf{K})$ is well-defined.

Then by the intertwining properties (20) (21) we have the following for each $\lambda$ and $\nu$,

$$
\begin{aligned}
& \sum_{\mu} \check{L}(\mathbf{L})_{\lambda}^{\mu} \otimes \check{L}(\mathbf{K})_{\mu}^{\nu} \check{R}^{\mathbf{K}, \mathbf{L}} \\
& =\sum_{\mu}\left(\phi_{\lambda \mathbf{L}}^{\mu} \phi_{\lambda}^{\mu \mathbf{L}}\right) \otimes\left(\phi_{\mu \mathbf{K}}^{\nu} \phi_{\mu}^{\nu \mathbf{K}}\right) \check{R}^{\mathbf{K}, \mathbf{L}} \\
& =\sum_{\mu}\left(\phi_{\lambda \mathbf{L}}^{\mu} \otimes \phi_{\mu \mathbf{K}}^{\nu}\right)\left(\phi_{\lambda}^{\mu \mathbf{L}} \otimes \phi_{\mu}^{\nu \mathbf{K}}\right) \check{R}^{\mathbf{K}, \mathbf{L}} \\
& =\sum_{\mu} \phi_{\lambda \mathbf{L}}^{\mu} \otimes \phi_{\mu \mathbf{K}}^{\nu} \sum_{\mu^{\prime}} \check{W}^{\mathbf{K L}}\left[\begin{array}{ccc} 
& \mu^{\prime} & \\
\lambda & & \nu \\
& &
\end{array}\right] \phi_{\lambda}^{\mu^{\prime} \mathbf{K}} \otimes \phi_{\mu^{\prime}}^{\nu \mathbf{L}} \\
& =\check{R}^{\mathbf{K}, \mathbf{L}} \sum_{\mu^{\prime}}\left(\phi_{\lambda \mathbf{K}}^{\mu^{\prime}} \otimes \phi_{\mu^{\prime} \mathbf{L}}^{\nu}\right)\left(\phi_{\lambda}^{\mu^{\prime} \mathbf{K}} \otimes \phi_{\mu^{\prime}}^{\nu \mathbf{L}}\right) \\
& =\check{R}^{\mathbf{K}, \mathbf{L}} \sum_{\mu^{\prime}}\left(\phi_{\lambda \mathbf{K}}^{\mu^{\prime}} \phi_{\lambda}^{\mu^{\prime} \mathbf{K}}\right) \otimes\left(\phi_{\mu^{\prime} \mathbf{L}}^{\nu} \phi_{\mu^{\prime}}^{\nu \mathbf{L}}\right) \\
& =\check{R}^{\mathbf{K}, \mathbf{L}} \sum_{\mu^{\prime}} \check{L}(\mathbf{K})_{\lambda}^{\mu^{\prime}} \otimes \check{L}(\mathbf{L})_{\mu^{\prime}}^{\nu}
\end{aligned}
$$


This identity of operators $V(\mathbf{K}) \otimes V(\mathbf{L}) \rightarrow V(\mathbf{L}) \otimes V(\mathbf{K})$ implies the assertion.
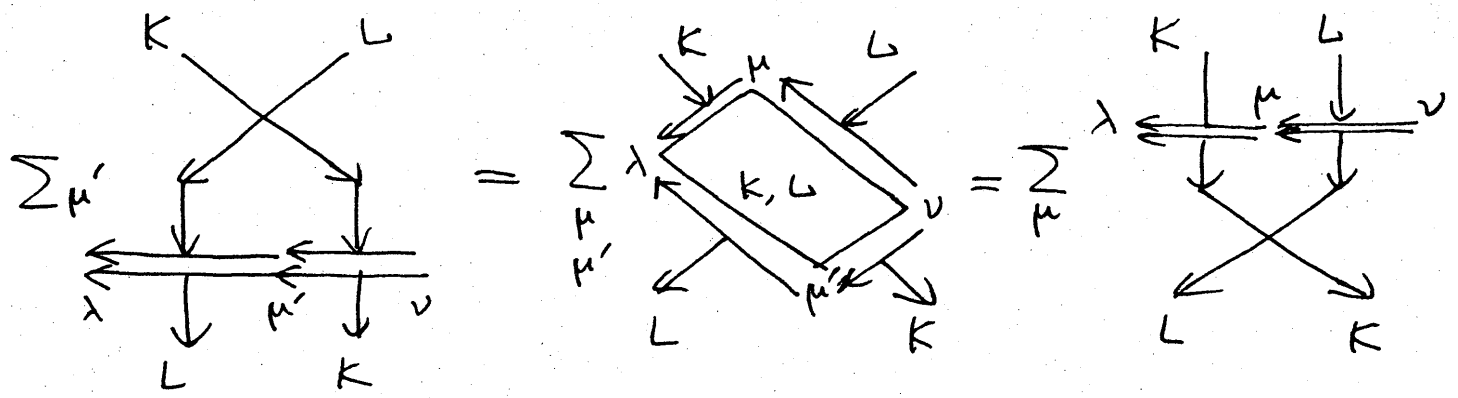

Remark. Recall the definition $V\left(1_{u}^{k}\right):=\pi_{1^{k}}\left(V\left(\square_{u}\right) \otimes \cdots \otimes V\left(\square_{u+k \hbar}\right)\right)$, where $V\left(\square_{u}\right)$ is just a copy of $\mathbf{C}^{n}$ (section 3$)$. This implies $V\left(1_{u+x}^{k}\right) \cong V\left(1_{u}^{k}\right)$. Similarly $\mathcal{P}_{\lambda 1_{u+x}^{k}}^{\mu} \cong \mathcal{P}_{\lambda 1_{u}^{k}}^{\mu}$. So identify these spaces and denote them as $V\left(1^{k}\right), \mathcal{P}_{\lambda 1^{k}}^{\mu}$ respectively. Then we have the operator

$$
\begin{aligned}
\check{L}\left(1_{u+x}^{k}, 1_{u}^{k}\right)_{\lambda}^{\mu}:= & \phi_{\lambda 1_{u+x}^{k}}^{\mu} \phi_{\lambda}^{\mu 1_{u}^{k}} \\
: & V\left(1^{k}\right) \cong V\left(1_{u}^{k}\right) \rightarrow \mathcal{P}_{\lambda 1_{u}^{k}}^{\mu} \cong \mathcal{P}_{\lambda 1_{u+x}^{k}}^{\mu} \rightarrow V\left(1_{u+x}^{k}\right) \cong V\left(1^{k}\right)
\end{aligned}
$$

and we can define $\check{L}\left(1_{u+x}^{k}, 1_{u}^{k}\right)$ by $\check{L}\left(1_{u+x}^{k}, 1_{u}^{k}\right)\left(\mathrm{v} \otimes \delta^{\mu}\right):=\sum_{\lambda} \delta^{\lambda} \otimes \check{L}\left(1_{u+x}^{k}, 1_{u}^{k}\right)_{\lambda}^{\mu}(\mathrm{v})$ - Adapting the above identification of spaces we can say that the operators $\check{R}^{1_{u}^{k}, 1_{v}^{l}}$ and $\check{W}^{1_{u}^{k}, 1_{v}^{l}}$ depends only on their deference $u-v$. Then we apply the above proof and get

$$
\begin{aligned}
& \left(\check{L}\left(\mathbf{L}_{+x}, \mathbf{L}\right) \otimes 1\right)\left(1 \otimes \check{L}\left(\mathbf{K}_{+x}, \mathbf{K}\right)\right)\left(\check{R}^{\mathbf{K}, \mathbf{L}} \otimes 1\right) \\
= & \left(1 \otimes \check{R}^{\mathbf{K}, \mathbf{L}}\right)\left(1 \otimes \check{L}\left(\mathbf{K}_{+x}, \mathbf{K}\right)\right)\left(\check{L}\left(\mathbf{L}_{+x}, \mathbf{L}\right) \otimes 1\right),
\end{aligned}
$$

where $\mathbf{K}=1_{u}^{k}, \mathbf{K}_{+x}=1_{u+x}^{k}, \mathbf{L}=1_{v}^{l}, \mathbf{L}_{+x}=1_{v+x}^{l}$.

The $\mathrm{L}$ operator given in this section defines a representation of the algebra of L-operators [KS] on $\mathcal{V}(24)$. This is rather a large space but contains some series of sub/quotient representations. First, we can restrict (the contragradient of) this representation to the space of quasi doubly periodic meromorphic 
functions on the weight space $\mathbf{h}^{*}$. This can be considered as a generalization of series a) in Sklyanin's work [S]. Second, letting $\hbar$ to be a rational number we get a "cyclic" representations from this, which generalize the series b) in [S], and suggest the generalization of the Kashiwara-Miwa's solution of the star-triangle equation. We hope to discuss these important aspects of our L-operator elsewhere.

Acknowledgement. The author expresses his gratitude to Professor G.Kuroki for fruitful disscussions, and Professor R.Hotta, Professor M.Jimbo and Professor A.Tsuchiya for their kind encouragements.

\section{References}

[AMPTY] H.Au-Yang, B.M.McCoy, J.H.H.Perk,S.Tang and M.-L.Yan, " Commuting transfer matrices in the chiral Potts models: solutions of star-triangle equations with genus $>1$ ", Phys. Lett. A 123, 219-223, 219-223.

[Bax] R.J.Baxter, Exactly solved models in statistical mechanics, Academic, London (1972).

[Bax73] R.J.Baxter, "Eight-vertex model in lattice statistics and one-dimensional anisotropic Heisenberg chain." I.Ann. Phys. 76 (1973) 1-24, II. ibid. 2547, III. ibid. 48-71.

[BPA] R.J.Baxter, J.H.H.Perk and H.Au-Yang "New solutions of the startriangle relations for the chiral Potts model " , Phys. Lett. A 128, 138-142 (1988).

[Be] A.A.Belavin, "Dynamical symmetry of.integrable quantum systems", Nucl. Phys. B180 [FS2] 189-200 (1981).

[BS] V.V.Bazhanov and Yu.G.Stroganov, "Chiral Potts model as a descendant of the six vertex model", J. Stat. Phys. 59, 799-817 (1990). 
[BKMS] V.V.Bazhanov, R.M.Kashaev, V.V.Mangazeev and Yu.G.Stroganov, " $\left(\mathbf{Z}_{N} \times\right)^{n-1}$ generalizaton of the Chiral Potts model", Comm. Math. Phys. 138, 393-408(1991).

[C] I.V.Cherednik, "Some finite dimensional representation of generalized Sklyanin algebras", Funct. Annal. and Appl. (Engl. transl.) 19, 77-79 (1985) ; "On "quantum" deformations of irreducible finite-dimensional representations of $g l_{N} "$, Sov. Math. Dokl. (Engl. transl.) 33, 507-510 (1986)

[FR] I.B.Frenkel and N.Yu.Reshetikhin, "Quantum affine algebras and holonomic difference equations", Comm. Math. Phys. 146, 1-60 (1992).

[H] K.Hasegawa, "On the crossing symmetry of the broken $Z_{N}$ symmetric solution of the Yang-Baxter equation", to appear in the proceedings of the ICM satelite at Kawaguchi-ko on representation theory, World Scientific.

[HY] K.Hasegawa and Y.Yamada, "Algebraic Derivation of the broken $\mathbf{Z}_{N^{-}}$ Symmetric model", Phys. Lett. A146, 387-396 (1990).

[IK] A,G.Izerin and V.E.Korepin, Lattice version of quantum field theory models in two dimensions, Nucl. Phys. B205 [FS5] 401-413 (1982).

[J] M.Jimbo, "A q-difference analog of $U(g)$ and the Yang-Baxter equation", Lett. math. Phys.10, 63-69 (1985).

[JMO] M.Jimbo, T.Miwa and M.Okado, "Solvable lattice models whose states are dominant integral weights of $A_{n-1}^{(1)}$ ", Lett. Math. Phys. 14, 123-131 (1987) ; "Local state probabilities of solvable lattice models: An $A_{n}^{(1)}$ family", Nucl. Phys. B300 [FS22] 74-108 (1988).

[JKMO] M.Jimbo, A.Kuniba, T.Miwa and M.Okado, "The $A_{n}^{(1)}$ Face Models", Comm. Math. Phys., 119 543-565 (1988). 
[KM] M.Kashiwara and T.Miwa, "A class of elliptic solutions to the startriangle relation", Nucl. Phys. B275 [FS17], 121-134 (1986).

[KS] P.P.Kulish and E.K.Sklyanin, "Quantum spectral transform method. Rescent developments", Lecture Notes in Physics 151, 61-119, Springer (1982).

[KRS] P.P.Kulish,N.Yu.Reshetikhin and E.K.Sklyanin, "Yang-Baxter equation and representation theory I", Lett. math. phys. 5, 393-403(1981).

[RT] M.P.Richey and C.A.Tracy, " $\mathbf{Z}_{n}$ Baxter model: symmetries and the Belavin parametrization", J. Stat. Phys. 42, 311-348 (1986).

[S] E.K.Sklyanin, "Some algebraic structure connected with the YangBaxter equation", Funct. Anal. and Appl. (Engl. transl.) 16, 27-34 (1982); 17, 273-284 (1983). 